\title{
Soliton dynamics of NLS with singular potentials
}

\author{
Claudio Bonanno, Marco Ghimenti, and Marco Squassina
}

Communicated by Y. Charles Li, received March 5, 2013.

\begin{abstract}
We investigate the validity of a soliton dynamics behavior in the semi-classical limit for the nonlinear Schrödinger equation in $\mathbb{R}^{N}, N \geq 3$, in presence of a singular external potential.
\end{abstract}

\section{Contents}

1. Introduction and main result 177

2. Some preparatory results $\quad 180$

3. Intermediate proofs 184

4. Proof of the main result completed 198

$\begin{array}{lll}\text { Appendix A. Semi-singular potentials } & 198\end{array}$

References 206

\section{Introduction and main result}

For $\varepsilon \in(0,1], N \geq 3$ and $0<p<2 / N$, we consider the nonlinear Schrödinger equation

$$
\imath \varepsilon \partial_{t} u_{\varepsilon}+\frac{\varepsilon^{2}}{2} \Delta u_{\varepsilon}-V(x) u_{\varepsilon}+\left|u_{\varepsilon}\right|^{2 p} u_{\varepsilon}=0, \quad t>0, x \in \mathbb{R}^{N}
$$

in presence of a real external potential $V$. This equation typically appears for the propagation of light in nonlinear optical materials which exhibit some kind of inhomogeneities, see [20] and the references therein for more details. For a smooth potential $V$, the problem of orbital stability of standing wave solutions to (1.1) has been extensively studied, see e.g. $[\mathbf{5}, \mathbf{9}, \mathbf{1 0}]$. Beside some studies of $(1.1)$ in the

2000 Mathematics Subject Classification. 35D99, 35J62, 58E05, 35J70.

Key words and phrases. Nonlinear Schrödinger equation, soliton dynamics, singular potentials.

The authors were partially supported by the MIUR projects PRIN2009: "Variational and Topological Methods in the Study of Nonlinear Phenomena" and "Critical Point Theory and Perturbative Methods for Nonlinear Differential Equations". 
framework of geometric optics and via suitable perturbation methods [5], several contributions appeared on the rigorous derivation of the soliton dynamics behavior in the semi-classical limit $\varepsilon \rightarrow 0$ for (1.1) with bump-like initial data. Essentially, two rather different approaches are currently available in the literature. On one hand, the seminal paper by Bronski and Jerrard [8], refined by [14], adopted a technique which includes a combination of quantum and classical conservation laws with the modulational stability property of ground states due to Weinstein $[\mathbf{2 1}, \mathbf{2 2}]$, see $[\mathbf{6}, \mathbf{7}, \mathbf{1 4}]$ and the references therein. On the other hand a different and more geometrical approach was developed in a series of papers $[\mathbf{3}, \mathbf{1 1}-\mathbf{1 3}]$. Subsequently, based on the first approach, further developments were achieved for a class of weakly coupled Schrödinger systems $[\mathbf{1 6}, \mathbf{1 7}]$ as well as for equations with an external electromagnetic field $[\mathbf{1 8}, \mathbf{1 9}]$. In all of these manuscripts, the external potential $V$ is always assumed to be a smooth function on $\mathbb{R}^{N}$ with bounded derivatives up to order three. For rough and time-dependent potentials see $[\mathbf{1}, \mathbf{2}]$.

In this paper, we shall derive a soliton dynamics behavior for (1.1) in presence of a smooth but singular potential. To our knowledge, previous contributions on this case consider the one dimensional case, see e.g. [4] and the references therein. We shall assume that $V$ satisfies the following conditions:

(V1) $V \in C^{\infty}\left(\mathbb{R}^{N} \backslash\{0\}, \mathbb{R}\right)$ is such that

$$
V(x) \sim|x|^{-\beta}, \quad|\nabla V(x)| \lesssim|x|^{-(\beta+1)}, \quad|\nabla| \nabla V|(x)| \lesssim|x|^{-(\beta+2)}, \quad \text { as }|x| \rightarrow 0
$$

where $0<\beta<1$;

(V2) $V(x) \geq V_{0}=\inf _{\mathbb{R}^{N}} V>0$ for all $x \in \mathbb{R}^{N} \backslash\{0\}$ and $\frac{|\nabla V(x)|^{2}}{\sqrt{V(x)-V_{0}}} \in L^{N}\left(\mathbb{R}^{N} \backslash\right.$ $B(0,1))$;

(V3) for each $\delta>0$ it holds $\phi(\delta)<+\infty$, where $\phi:(0, \infty) \rightarrow(0, \infty)$ is defined by

$$
\phi(\delta):=\sum_{|\alpha|=0}^{3}\left\|D^{\alpha} V\right\|_{L^{\infty}\left(B_{\delta}\right)}, \quad B_{\delta}:=\mathbb{R}^{N} \backslash B(0, \delta) .
$$

Hence $V$ is bounded away from zero and has only one singularity located, with no loss of generality, at the origin and is elsewhere smooth and uniformly bounded together with the higher order derivatives up to the order three. Next, we introduce the initial conditions to be assigned to equation (1.1). Let $H$ denote the energy space, that is $H^{1}\left(\mathbb{R}^{N}\right)$ endowed with the standard norm

$$
\|u\|_{H}^{2}:=\int_{\mathbb{R}^{N}}\left(|\nabla u|^{2}+|u|^{2}\right) .
$$

We also introduce the $H_{\varepsilon}^{1}$-norm defined on $H$ as

$$
\|u\|_{H_{\varepsilon}^{1}}^{2}:=\frac{1}{\varepsilon^{N-2}} \int_{\mathbb{R}^{N}}|\nabla u|^{2}+\frac{1}{\varepsilon^{N}} \int_{\mathbb{R}^{N}}|u|^{2}, \quad u \in H .
$$

Let $R$ be the positive radial solution to

$$
-\frac{1}{2} \Delta R(x)+R(x)=R(x)^{2 p+1}, \quad x \in \mathbb{R}^{N} .
$$


It is well known that $R$ is unique (up to translations) [15] and exponentially decaying, satisfying

$$
\lim _{|x| \rightarrow+\infty} R(x)|x|^{\frac{N-1}{2}} e^{|x|}=\text { const. }
$$

Moreover let $\left(x_{0}, \xi_{0}\right) \in \mathbb{R}^{N} \times \mathbb{R}^{N}$ with $x_{0} \neq 0$. It is readily seen that there exists $\delta=\delta\left(x_{0}, \xi_{0}\right)>0$ such that the solution $(x(t), \xi(t))$ to the Newtonian system

$$
\left\{\begin{array}{l}
\dot{x}=\xi \\
\dot{\xi}=-\nabla V(x) \\
x(0)=x_{0} \\
\xi(0)=\xi_{0}
\end{array}\right.
$$

is global in time and satisfies

$$
\inf _{t}|x(t)|>\delta, \quad \sup _{t}|\xi(t)|<\sqrt{\left|\xi_{0}\right|^{2}+2 V\left(x_{0}\right)} .
$$

This easily follows by the Hamiltonian function for (1.6), given by

$$
\mathcal{H}(x, \xi)=\frac{1}{2}|\xi|^{2}+V(x), \quad x, \xi \in \mathbb{R}^{N} .
$$

Let $v_{\varepsilon}(x)$ be a function satisfying:

(C1) $v_{\varepsilon}(x) \in H$ and is radially symmetric with respect to $x_{0}$;

(C2) there exist $\gamma>0$ and $\left(x_{0}, \xi_{0}\right) \in \mathbb{R}^{N} \times \mathbb{R}^{N}$ with $x_{0} \neq 0$ such that

$$
\left\|v_{\varepsilon}(x)-R\left(\frac{x-x_{0}}{\varepsilon}\right) e^{e^{\frac{\xi_{0} \cdot x}{\varepsilon}}}\right\|_{H_{\varepsilon}^{1}}^{2}<\gamma
$$

(C3) for $\delta=\delta\left(x_{0}, \xi_{0}\right)>0$ as defined in (1.7), there exists $\rho \in\left(0,\left|x_{0}\right|-\delta\right)$ such that

(C4) $\frac{1}{\varepsilon^{N}}\left\|v_{\varepsilon}(x)\right\|_{L^{2}}^{2}=\|R\|_{L^{2}}^{2}=: m$.

$$
\operatorname{supp} v_{\varepsilon}(x) \subset B\left(x_{0}, \rho\right) ;
$$

We are then reduced to study the initial value problem

$$
\left\{\begin{array}{l}
\imath \varepsilon \partial_{t} u_{\varepsilon}+\frac{\varepsilon^{2}}{2} \Delta u_{\varepsilon}-V(x) u_{\varepsilon}+\left|u_{\varepsilon}\right|^{2 p} u_{\varepsilon}=0, \\
u_{\varepsilon} \in H, \\
u_{\varepsilon}(0, x)=v_{\varepsilon}(x),
\end{array}\right.
$$

where $V$ satisfies (V1)-(V3) and the initial datum $v_{\varepsilon}$ satisfies $(\mathrm{C} 1)-(\mathrm{C} 4)$. Under the above assumptions, (1.9) admits a global strong solution, that is a function

$$
u_{\varepsilon} \in C^{0}(\mathbb{R}, H) \cap C^{1}\left(\mathbb{R}, H^{-1}\right),
$$

such that $u_{\varepsilon}(0, x)=v_{\varepsilon}(x)$ and, for all $C_{0}^{\infty}\left(\mathbb{R}^{N}, \mathbb{C}\right)$ and $t>0$

$$
\begin{aligned}
& \Re \int_{\mathbb{R}^{N}} \imath \varepsilon \partial_{t} u_{\varepsilon}(t, x) \bar{\varphi}(t, x)-\frac{\varepsilon^{2}}{2} \nabla u_{\varepsilon}(t, x) \cdot \nabla \bar{\varphi}(t, x) \\
& -V(x) u_{\varepsilon}(t, x) \bar{\varphi}(t, x)+\left|u_{\varepsilon}(t, x)\right|^{2 p} u_{\varepsilon}(t, x) \bar{\varphi}(t, x)=0 .
\end{aligned}
$$

Furthermore, there holds $u_{\varepsilon}(t) \in H^{2}\left(\mathbb{R}^{N}\right)$ and $\partial_{t} u_{\varepsilon}(t) \in L^{2}\left(\mathbb{R}^{N}\right)$, for all $t>0$. Since under our assumptions $V \in L^{m}\left(\mathbb{R}^{N}\right)+L^{\infty}\left(\mathbb{R}^{N}\right)$ for $m \geq 2$ with $m>N / 2$, this holds true in light of [9, see Theorem 4.3.1 and Remark 4.3.2 for local wellposedness and conservation laws as well as Theorem 5.2.1 and Remark 5.2.9 for 
the regularity $\left.H^{2}\left(\mathbb{R}^{N}\right)\right]$ jointly with the a priori estimate for all $t>0$ obtained in Lemma 2.3.

To our knowledge, the following result is the first attempt to describe the soliton dynamics in presence of a singular potential in several dimensions. Under the previous assumptions it holds

THEOREM 1.1. Assume that, for a small $\varepsilon>0$, we have

$$
\gamma \leq \varepsilon^{4 \frac{17+\beta}{1-\beta}}, \quad\left|\xi_{0}\right| \leq \varepsilon^{\frac{17+\beta}{1-\beta}}, \quad \int_{B\left(x_{0}, \rho\right)}\left(V(x)-V_{0}\right)\left|v_{\varepsilon}(x)\right|^{2} \leq \varepsilon^{N+2 \frac{17+\beta}{1-\beta}}
$$

Then there exists a map $\theta_{\varepsilon}: \mathbb{R}^{+} \rightarrow[0,2 \pi)$ such that

$$
u_{\varepsilon}(x, t)=R\left(\frac{x-x(t)}{\varepsilon}\right) e^{\frac{\imath}{\varepsilon}\left[x \cdot \xi(t)+\theta_{\varepsilon}(t)\right]}+\omega_{\varepsilon}(x, t),
$$

with $\left\|\omega_{\varepsilon}(\cdot, t)\right\|_{H_{\varepsilon}^{1}}=O(\varepsilon)$, as $\varepsilon \rightarrow 0$, locally uniformly in time.

Roughly speaking, in order to preserve the shape of the initial profile and to describe the dynamics, one has to start with a bump-like initial data located sufficiently far from the singularity and with a small enough initial velocity. Precisely, for the model potential $V(x)=|x|^{-\beta}$ one should assume that $\left|x_{0}\right| \geq 2 / \varepsilon^{2(17+\beta) /\left(\beta-\beta^{2}\right)}$ in order to fulfill the last inequality of assumption (1.10).

The result is proved by arguments in the spirit of [8]. On the other hand, the presence of the singular potential requires a very careful analysis and new subtle estimates have to be established. In particular, we refer the reader to Propositions 3.6 and 3.7.

Finally, in Appendix A we shall provide the estimates related with the soliton dynamics when the singular potential is truncated around the singularity. We believe that this can be useful, especially for numerical purposes.

Throughout the manuscript we shall always give the explicit dependence of the constants involved in the estimates. The constants will often depend on the initial conditions $\left(x_{0}, \xi_{0}, v_{\varepsilon}\right)$ but in a uniform manner with respect to $\varepsilon$. That is, let $\varepsilon_{0}$ be such that Theorem 1.1 holds for $\varepsilon<\varepsilon_{0}$. Then the different constants $\operatorname{const}\left(x_{0}, \xi_{0}, v_{\varepsilon}\right)$ in the following can be bounded by $\operatorname{const}\left(x_{0}, \xi_{0}, v_{\varepsilon_{0}}\right)$.

\section{Some preparatory results}

Using the variational structure of (1.1), it is readily checked that the solution $u_{\varepsilon}$ satisfies

$$
\begin{gathered}
\frac{d}{d t} \frac{1}{\varepsilon^{N}}\left|u_{\varepsilon}(t, x)\right|^{2}=-\nabla \cdot p_{\varepsilon}(t, x), \quad t>0, \quad x \in \mathbb{R}^{N}, \\
\frac{d}{d t} \int_{\mathbb{R}^{N}} p_{\varepsilon}(t, x)=-\int_{\mathbb{R}^{N}} \frac{1}{\varepsilon^{N}}\left|u_{\varepsilon}(t, x)\right|^{2} \nabla V(x), \quad t>0,
\end{gathered}
$$

where

$$
p_{\varepsilon}(t, x):=\frac{1}{\varepsilon^{N-1}} \Im\left(\overline{u_{\varepsilon}}(t, x) \nabla u_{\varepsilon}(t, x)\right), \quad(t, x) \in \mathbb{R} \times \mathbb{R}^{N},
$$

where $\Im(z)$ denotes the imaginary part of the complex number $z$. 
Both side terms are finite by assumptions on $u_{\varepsilon}$ and (V1) since $|\nabla V| \in L^{N / 2}\left(\mathbb{R}^{N}\right)$. Notice that, equation (2.1) implies the conservation of mass, for every $\varepsilon>0$,

$$
m:=\frac{1}{\varepsilon^{N}} \int_{\mathbb{R}^{N}}\left|u_{\varepsilon}(t, x)\right|^{2}=\frac{1}{\varepsilon^{N}} \int_{\mathbb{R}^{N}}\left|v_{\varepsilon}(x)\right|^{2},
$$

and equation (2.2) gives the evolution law for the momentum

$$
P_{\varepsilon}\left(u_{\varepsilon}, t\right):=\int_{\mathbb{R}^{N}} p_{\varepsilon}(t, x) .
$$

For a global strong solution to (1.9) the energy defined as follows, is conserved

$$
\begin{aligned}
E_{\varepsilon}\left(u_{\varepsilon}, t\right):= & \frac{1}{2 \varepsilon^{N-2}} \int_{\mathbb{R}^{N}}\left|\nabla u_{\varepsilon}(t, x)\right|^{2}-\frac{1}{(p+1) \varepsilon^{N}} \int_{\mathbb{R}^{N}}\left|u_{\varepsilon}(t, x)\right|^{2 p+2} \\
& +\frac{1}{\varepsilon^{N}} \int_{\mathbb{R}^{N}} V(x)\left|u_{\varepsilon}(t, x)\right|^{2}
\end{aligned}
$$

We recall that the function $R$ is a point of minimum for the energy

$$
\mathscr{E}(v):=\frac{1}{2} \int_{\mathbb{R}^{N}}|\nabla v(x)|^{2}-\frac{1}{p+1} \int_{\mathbb{R}^{N}}|v(x)|^{2 p+2},
$$

constrained to the manifold of functions in $H^{1}\left(\mathbb{R}^{N}\right)$ with fixed $L^{2}$-norm equal to $\sqrt{m}$. Let us denote

$$
\mathscr{E}_{\varepsilon}(v):=\frac{1}{2 \varepsilon^{N-2}} \int_{\mathbb{R}^{N}}|\nabla v(x)|^{2}-\frac{1}{(p+1) \varepsilon^{N}} \int_{\mathbb{R}^{N}}|v(x)|^{2 p+2} .
$$

Then, we have the following

LEMMA 2.1. There exist $\gamma_{0}>0$ such that, if $v_{\varepsilon}$ satisfies assumptions $(\mathrm{C} 1)-(\mathrm{C} 4)$ for any $\varepsilon>0$ small enough and any $\gamma \in\left(0, \gamma_{0}\right)$, then there exists a positive constant merely depending on $R$ and $\xi_{0}$ such that

$$
\left|\mathscr{E}_{\varepsilon}\left(v_{\varepsilon}\right)-\mathscr{E}_{\varepsilon}\left(R\left(\frac{x-x_{0}}{\varepsilon}\right) e^{\imath} \frac{\xi_{0} \cdot x}{\varepsilon}\right)\right| \leq \operatorname{const}\left(R, \xi_{0}\right) \sqrt{\gamma} .
$$

Proof. We shall use the elementary inequality

$$
\forall \zeta>0 \quad \exists C_{\zeta}>0: \quad|| a+\left.b\right|^{r}-\left.|b|^{r}|\leq \zeta| b\right|^{r}+C_{\zeta}|a|^{r},
$$

for all $a, b \in \mathbb{C}$ and $r \in(1, \infty)$, where $C_{\zeta}$ blows up as $\zeta^{1-r}$ as $\zeta$ goes to zero. Indeed, we first write

$$
\begin{aligned}
& \left.\left|\frac{1}{\varepsilon^{N-2}} \int_{\mathbb{R}^{N}}\right| \nabla v_{\varepsilon}(x)\right|^{2}-\frac{1}{\varepsilon^{N-2}} \int_{\mathbb{R}^{N}}\left|\nabla\left[R\left(\frac{x-x_{0}}{\varepsilon}\right) e^{\imath \frac{\xi_{0} \cdot x}{\varepsilon}}\right]\right|^{2} \mid \\
& \leq \frac{\zeta}{\varepsilon^{N-2}} \int_{\mathbb{R}^{N}}\left|\nabla\left[R\left(\frac{x-x_{0}}{\varepsilon}\right) e^{\imath \frac{\xi_{0} \cdot x}{\varepsilon}}\right]\right|^{2}+C_{\zeta}\left\|v_{\varepsilon}(x)-R\left(\frac{x-x_{0}}{\varepsilon}\right) e^{\imath \frac{\xi_{0} \cdot x}{\varepsilon}}\right\|_{H_{\varepsilon}^{1}}^{2} \\
& \leq \zeta\left(\int_{\mathbb{R}^{N}}|\nabla R|^{2}+m\left|\xi_{0}\right|^{2}\right)+C_{\zeta} \gamma=O(\sqrt{\gamma}),
\end{aligned}
$$

after choosing $\zeta=\sqrt{\gamma}$ and using the asymptotics $C_{\zeta} \sim \gamma^{-1 / 2}$ for small $\gamma$. The constant in $O(\sqrt{\gamma})$ depends only on $R$ and $\xi_{0}$. Concerning the second term in the energy $\mathscr{E}_{\varepsilon}$, we get

$$
\begin{aligned}
& \left.\left|\frac{1}{\varepsilon^{N}} \int_{\mathbb{R}^{N}}\right| v_{\varepsilon}(x)\right|^{2 p+2}-\frac{1}{\varepsilon^{N}} \int_{\mathbb{R}^{N}}\left|R\left(\frac{x-x_{0}}{\varepsilon}\right) e^{\imath \frac{\xi_{0} \cdot x}{\varepsilon}}\right|^{2 p+2} \mid \\
& \leq \frac{\zeta}{\varepsilon^{N}} \int_{\mathbb{R}^{N}}\left|R\left(\frac{x-x_{0}}{\varepsilon}\right)\right|^{2 p+2}+\frac{C_{\zeta}}{\varepsilon^{N}} \int_{\mathbb{R}^{N}}\left|v_{\varepsilon}(x)-R\left(\frac{x-x_{0}}{\varepsilon}\right) e^{\imath \frac{\xi_{0} \cdot x}{\varepsilon}}\right|^{2 p+2}
\end{aligned}
$$


By the Gagliardo-Nirenberg inequality

$$
\|v\|_{L^{q}} \leq \operatorname{const}(q)\|v\|_{L^{2}}^{1-\frac{N}{2}+\frac{N}{q}}\|\nabla v\|_{L^{2}}^{\frac{N}{2}-\frac{N}{q}}, \quad 2 \leq q \leq 2^{*},
$$

choosing $q=2 p+2$, in light of (C2) we obtain

$$
\begin{gathered}
\left.\left|\frac{1}{\varepsilon^{N}} \int_{\mathbb{R}^{N}}\right| v_{\varepsilon}(x)\right|^{2 p+2}-\frac{1}{\varepsilon^{N}} \int_{\mathbb{R}^{N}}\left|R\left(\frac{x-x_{0}}{\varepsilon}\right) e^{\imath \frac{\xi_{0} \cdot x}{\varepsilon}}\right|^{2 p+2} \mid \\
\leq \zeta\|R\|_{L^{2 p+2}}^{2 p+2}+C_{\zeta} \operatorname{const}(2 p+2) \gamma^{p+1}=O(\gamma),
\end{gathered}
$$

choosing $\zeta=\gamma$ and using $C_{\zeta} \sim \gamma^{1-p}$ as $\gamma \rightarrow 0$. Here the constant in $O(\gamma)$ depends only on $R$ and $p$.

LEMMA 2.2. If $v_{\varepsilon}$ satisfies assumptions (C1)-(C4) for any $\varepsilon>0$ and any $\gamma>0$, then there exists a positive constant only depending on $R, x_{0}$ and $\xi_{0}$ such that

$$
\left.\left|\frac{1}{\varepsilon^{N}} \int_{\mathbb{R}^{N}} V(x)\right| v_{\varepsilon}(x)\right|^{2}-m V\left(x_{0}\right) \mid \leq \operatorname{const}\left(R, x_{0}, \xi_{0}\right)\left(\gamma+\varepsilon^{2}\right) \phi(\delta),
$$

where $\phi$ is defined in (1.2) and $\delta=\delta\left(x_{0}, \xi_{0}\right)$ is defined in (1.7).

Proof. We write

$$
\begin{aligned}
& \frac{1}{\varepsilon^{N}} \int_{\mathbb{R}^{N}} V(x)\left|v_{\varepsilon}(x)\right|^{2} \\
& =\frac{1}{\varepsilon^{N}} \int_{B\left(x_{0}, \rho\right)} V(x)\left|v_{\varepsilon}(x)\right|^{2}=\int_{B\left(0, \frac{\rho}{\varepsilon}\right)} V\left(x_{0}+\varepsilon y\right)\left|v_{\varepsilon}\left(x_{0}+\varepsilon y\right)\right|^{2} \\
& =\int_{B\left(0, \frac{\rho}{\varepsilon}\right)} V\left(x_{0}\right)\left|v_{\varepsilon}\left(x_{0}+\varepsilon y\right)\right|^{2}+\int_{B\left(0, \frac{\rho}{\varepsilon}\right)} \varepsilon\left(\nabla V\left(x_{0}\right) \cdot y\right)\left|v_{\varepsilon}\left(x_{0}+\varepsilon y\right)\right|^{2} \\
& +\int_{B\left(0, \frac{\rho}{\varepsilon}\right)} \varepsilon^{2}\left(\nabla^{2} V\left(x_{0}+\varepsilon \omega_{\varepsilon}(y) y\right) y \cdot y\right)\left|v_{\varepsilon}\left(x_{0}+\varepsilon y\right)\right|^{2} \\
& \leq m V\left(x_{0}\right)+O\left(\varepsilon^{2} \phi(\delta)\right) \int_{B\left(0, \frac{\rho}{\varepsilon}\right)}|y|^{2}\left|v_{\varepsilon}\left(x_{0}+\varepsilon y\right)\right|^{2},
\end{aligned}
$$

for some $\omega_{\varepsilon}(y) \in(0,1)$, where we have used the radial symmetry of $v_{\varepsilon}(x)$, the definition of $\phi(\delta)$ in (1.2) and assumptions (V3) and (C4). Moreover, we also have

$$
\begin{aligned}
& \int_{B\left(0, \frac{\rho}{\varepsilon}\right)}|y|^{2}\left|v_{\varepsilon}\left(x_{0}+\varepsilon y\right)\right|^{2} \\
& \leq 2 \int_{B\left(0, \frac{\rho}{\varepsilon}\right)}|y|^{2}\left|v_{\varepsilon}\left(x_{0}+\varepsilon y\right)-R(y) e^{\frac{\imath}{\varepsilon} \xi_{0} \cdot\left(x_{0}+\varepsilon y\right)}\right|^{2}+2 \int_{B\left(0, \frac{\rho}{\varepsilon}\right)}|y|^{2}|R(y)|^{2} \\
& \leq \frac{2 \rho^{2}}{\varepsilon^{2}}\left\|v_{\varepsilon}(x)-R\left(\frac{x-x_{0}}{\varepsilon}\right) e^{\imath \frac{\xi_{0} \cdot x}{\varepsilon}}\right\|_{H_{\varepsilon}^{1}}^{2}+2 \int_{\mathbb{R}^{N}}|y|^{2}|R(y)|^{2}
\end{aligned}
$$

where the last integral is finite by virtue of (1.5).

We now state the following uniform bound for the $H_{\varepsilon}^{1}$-norm of solutions.

LEMma 2.3. Let $u_{\varepsilon}(t, x)$ be a global strong solution of problem (1.9). Then

$$
M\left(x_{0}, \xi_{0}, v_{\varepsilon}\right):=\sup _{t \in \mathbb{R}}\left\|u_{\varepsilon}(t, x)\right\|_{H_{\varepsilon}^{1}}<+\infty .
$$


Proof. By choosing $q=2 p+2$ in (2.7), by virtue of the conservation of mass, we obtain

$$
\begin{aligned}
& \left\|u_{\varepsilon}(t, \cdot)\right\|_{L^{2 p+2}}^{2 p+2} \\
& \leq \operatorname{const}(p)\left\|u_{\varepsilon}(t, \cdot)\right\|_{L^{2}}^{2\left(1-\frac{N}{2}+\frac{N}{2 p+2}\right)(p+1)}\left\|\nabla u_{\varepsilon}(t, \cdot)\right\|_{L^{2}}^{2\left(\frac{N}{2}-\frac{N}{2 p+2}\right)(p+1)} \\
& =\operatorname{const}(p) m^{1+p\left(1-\frac{N}{2}\right)}\left(\varepsilon^{N}\right)^{1+p\left(1-\frac{N}{2}\right)}\left(\frac{1}{\varepsilon^{N-2}}\left\|\nabla u_{\varepsilon}(t, \cdot)\right\|_{L^{2}}^{2}\right)^{\frac{p N}{2}}\left(\varepsilon^{N-2}\right)^{\frac{p N}{2}} \\
& =\operatorname{const}(p) m^{1+p\left(1-\frac{N}{2}\right)} \varepsilon^{N}\left(\frac{1}{\varepsilon^{N-2}}\left\|\nabla u_{\varepsilon}(t, \cdot)\right\|_{L^{2}}^{2}\right)^{\frac{p N}{2}}, \quad t>0
\end{aligned}
$$

In turn, since $p<\frac{2}{N}$, Young's inequality yields

$$
\frac{1}{(p+1) \varepsilon^{N}} \int_{\mathbb{R}^{N}}\left|u_{\varepsilon}(t, x)\right|^{2 p+2} \leq \frac{1}{4 \varepsilon^{N-2}} \int_{\mathbb{R}^{N}}\left|\nabla u_{\varepsilon}(t, x)\right|^{2}+\operatorname{const}(p), \quad t>0 .
$$

Therefore, by the conservation of energy, we can write

$$
E_{\varepsilon}\left(u_{\varepsilon}, 0\right)=E_{\varepsilon}\left(u_{\varepsilon}, t\right) \geq \frac{1}{4 \varepsilon^{N-2}} \int_{\mathbb{R}^{N}}\left|\nabla u_{\varepsilon}(t, x)\right|^{2}+\frac{V_{0}}{\varepsilon^{N}} \int_{\mathbb{R}^{N}}\left|u_{\varepsilon}(t, x)\right|^{2}-\operatorname{const}(p),
$$

and the thesis follows by $V_{0}>0$.

Remark 2.4. By virtue of Lemmas 2.1 and 2.2, the initial energy $E_{\varepsilon}\left(u_{\varepsilon}, 0\right)$ remains uniformly bounded with respect to $\varepsilon>0$. In turn, we have

$$
\sup _{\varepsilon>0} M\left(x_{0}, \xi_{0}, v_{\varepsilon}\right)<+\infty .
$$

Introducing now the radial notation

$$
u_{\varepsilon}(t, x)=\left|u_{\varepsilon}(t, x)\right| e^{i S_{\varepsilon}(t, x)}, \quad x \in \mathbb{R}^{N}, t>0,
$$

we write

$$
p_{\varepsilon}(t, x)=\frac{1}{\varepsilon^{N-1}}\left|u_{\varepsilon}(t, x)\right|^{2} \nabla S_{\varepsilon}(t, x), \quad x \in \mathbb{R}^{N}, t>0,
$$

for the momentum density, and the total energy $E_{\varepsilon}$ can be split into the sum

$$
E_{\varepsilon}\left(u_{\varepsilon}, t\right)=J_{\varepsilon}\left(u_{\varepsilon}, t\right)+K_{\varepsilon}\left(u_{\varepsilon}, t\right), \quad t>0,
$$

where $J_{\varepsilon}$ is the internal energy and it is defined as

$$
J_{\varepsilon}\left(u_{\varepsilon}, t\right):=\frac{1}{2 \varepsilon^{N-2}} \int_{\mathbb{R}^{N}}|\nabla| u_{\varepsilon}(t, x)||^{2}-\frac{1}{(p+1) \varepsilon^{N}} \int_{\mathbb{R}^{N}}\left|u_{\varepsilon}(t, x)\right|^{2 p+2}, \quad t>0,
$$

and $K_{\varepsilon}$ is the kinetic energy and it is defined as

$$
K_{\varepsilon}\left(u_{\varepsilon}, t\right):=\frac{1}{2 \varepsilon^{N-2}} \int_{\mathbb{R}^{N}}\left|u_{\varepsilon}(t, x)\right|^{2}\left|\nabla S_{\varepsilon}(t, x)\right|^{2}+\frac{1}{\varepsilon^{N}} \int_{\mathbb{R}^{N}} V(x)\left|u_{\varepsilon}(t, x)\right|^{2}, \quad t>0 .
$$

Then, we have the following

Proposition 2.5. There exist $\gamma_{0}>0$ such that for any $\varepsilon>0$ and any $\gamma \in$ $\left(0, \gamma_{0}\right)$, if $u_{\varepsilon}$ is a global strong solution of problem (1.9) with energy $E_{\varepsilon}$ as in formula (2.3), then there exists a constant depending only on $R, x_{0}$ and $\xi_{0}$ such that, for all $t>0$,

$$
\left|E_{\varepsilon}\left(u_{\varepsilon}, t\right)-\mathscr{E}(R)-m \mathcal{H}(x(t), \xi(t))\right| \leq \operatorname{const}\left(R, x_{0}, \xi_{0}\right)\left(\sqrt{\gamma}+\varepsilon^{2}\right) \phi(\delta),
$$


being $\phi$ defined in (1.2), $\delta=\delta\left(x_{0}, \xi_{0}\right)$ defined in (1.7), $\mathcal{H}$ the Hamiltonian function (1.8) and $(x(t), \xi(t))$ the solution to the Newtonian system (1.6). Furthermore,

$$
0 \leq K_{\varepsilon}\left(u_{\varepsilon}, t\right) \leq \frac{1}{2} m\left|\xi_{0}\right|^{2}+\frac{1}{\varepsilon^{N}} \int_{\mathbb{R}^{N}} V(x)\left|v_{\varepsilon}(x)\right|^{2}+\operatorname{const}\left(R, \xi_{0}\right) \sqrt{\gamma},
$$

for every $t>0$.

Proof. By the conservation of the energy $E_{\varepsilon}$ for solutions of (1.9), we can write

$$
E_{\varepsilon}\left(u_{\varepsilon}, t\right)=E_{\varepsilon}\left(u_{\varepsilon}, 0\right)=\mathscr{E}_{\varepsilon}\left(v_{\varepsilon}\right)+\frac{1}{\varepsilon^{N}} \int_{\mathbb{R}^{N}} V(x)\left|v_{\varepsilon}(x)\right|^{2}, \quad t>0
$$

Taking into account

$$
\mathscr{E}_{\varepsilon}\left(R\left(\frac{x-x_{0}}{\varepsilon}\right) e^{\imath \frac{\xi_{0} \cdot x}{\varepsilon}}\right)=\mathscr{E}(R)+\frac{1}{2} m\left|\xi_{0}\right|^{2}
$$

and that $\mathcal{H}(x(t), \xi(t))=\mathcal{H}\left(x_{0}, \xi_{0}\right)$ for all $t>0$ by the conservation of the Hamiltonian for (1.6), inequality (2.13) follows from Lemma 2.1 and Lemma 2.2. To prove (2.14), observe that since $\left\|u_{\varepsilon}(t, \varepsilon \cdot)\right\|_{L^{2}}^{2}=m$ for all $t>0$ and $R$ is a point of constrained minimum for $\mathscr{E}$ on the $L^{2}$ sphere or radius $\sqrt{m}$, we get

$$
\mathscr{E}(R) \leq \mathscr{E}\left(\left|u_{\varepsilon}(t, \varepsilon, \cdot)\right|\right)=J_{\varepsilon}\left(u_{\varepsilon}, t\right), \quad t>0
$$

Hence, we get

$$
\begin{aligned}
0 \leq K_{\varepsilon}\left(u_{\varepsilon}, t\right) & =E_{\varepsilon}\left(u_{\varepsilon}, t\right)-J_{\varepsilon}\left(u_{\varepsilon}, t\right) \leq E_{\varepsilon}\left(u_{\varepsilon}, 0\right)-\mathscr{E}(R) \\
& =\mathscr{E}_{\varepsilon}\left(v_{\varepsilon}(x)\right)+\frac{1}{\varepsilon^{N}} \int V(x)\left|v_{\varepsilon}(x)\right|^{2}-\mathscr{E}(R)-\frac{1}{2} m\left|\xi_{0}\right|^{2}+\frac{1}{2} m\left|\xi_{0}\right|^{2} \\
& \leq \frac{1}{2} m\left|\xi_{0}\right|^{2}+\frac{1}{\varepsilon^{N}} \int V(x)\left|v_{\varepsilon}(x)\right|^{2}+\operatorname{const}\left(R, \xi_{0}, p\right) \sqrt{\gamma}, \quad t>0
\end{aligned}
$$

by virtue of Lemma 2.1 .

\section{Intermediate proofs}

As in $[\mathbf{8}, \mathbf{1 4}]$, we introduce the auxiliary function

$$
\Psi^{\varepsilon}(t, x):=u_{\varepsilon}(t, x(t)+\varepsilon x) e^{-\frac{2}{\varepsilon} \xi(t) \cdot(x(t)+\varepsilon x)}, \quad x \in \mathbb{R}^{N}, t>0,
$$

which satisfies $\left\|\Psi^{\varepsilon}(t, \cdot)\right\|_{L^{2}}^{2}=m$ for all $t>0$. First of all we notice that

$$
\left\|\Psi^{\varepsilon}(0, \cdot)-R\right\|_{H^{1}}^{2} \leq\left(3+2\left|\xi_{0}\right|^{2}\right) \gamma
$$


which follows from simple computations. For the gradient term

$$
\begin{aligned}
& \int_{\mathbb{R}^{N}}\left|\nabla \Psi^{\varepsilon}(0, x)-\nabla R(x)\right|^{2} \\
& =\int_{\mathbb{R}^{N}}\left|\left(\varepsilon \nabla u_{\varepsilon}\left(0, \varepsilon x+x_{0}\right)-\imath u_{\varepsilon}\left(0, \varepsilon x+x_{0}\right) \xi_{0}\right) e^{-\frac{2}{\varepsilon} \xi_{0} \cdot\left(x_{0}+\varepsilon x\right)}-\nabla R(x)\right|^{2} \\
& =\frac{1}{\varepsilon^{N}} \int_{\mathbb{R}^{N}}\left|\left(\varepsilon \nabla v_{\varepsilon}(y)-\imath v_{\varepsilon}(y) \xi_{0}\right) e^{-\frac{2}{\varepsilon} \xi_{0} \cdot y}-\varepsilon \nabla\left[R\left(\frac{y-x_{0}}{\varepsilon}\right)\right]\right|^{2} \\
& =\frac{1}{\varepsilon^{N}} \int_{\mathbb{R}^{N}}\left|\varepsilon \nabla v_{\varepsilon}(y)-\imath v_{\varepsilon}(y) \xi_{0}-\varepsilon \nabla\left(R\left(\frac{y-x_{0}}{\varepsilon}\right) e^{\frac{2}{\varepsilon} \xi_{0} \cdot y}\right)+\imath R\left(\frac{y-x_{0}}{\varepsilon}\right) \xi_{0} e^{\frac{2}{\varepsilon} \xi_{0} \cdot y}\right|^{2} \\
& \leq \frac{2}{\varepsilon^{N}} \int_{\mathbb{R}^{N}}\left[\varepsilon^{2}\left|\nabla v_{\varepsilon}(y)-\nabla\left(R\left(\frac{y-x_{0}}{\varepsilon}\right) e^{\frac{2}{\varepsilon} \xi_{0} \cdot y}\right)\right|^{2}\right. \\
& \left.+\left|\xi_{0}\right|^{2}\left|v_{\varepsilon}(y)-R\left(\frac{y-x_{0}}{\varepsilon}\right) e^{\frac{2}{\varepsilon} \xi_{0} \cdot y}\right|^{2}\right] \\
& <2\left(1+\left|\xi_{0}\right|^{2}\right) \gamma,
\end{aligned}
$$

where in the last inequality we have used $(\mathrm{C} 2)$. For the $L^{2}$ term again

$$
\int_{\mathbb{R}^{N}}\left|\Psi^{\varepsilon}(0, x)-R(x)\right|^{2}=\frac{1}{\varepsilon^{N}} \int_{\mathbb{R}^{N}}\left|v_{\varepsilon}(y)-R\left(\frac{y-x_{0}}{\varepsilon}\right) e^{\frac{2}{\varepsilon} \xi_{0} \cdot y}\right|^{2}<\gamma,
$$

by virtue of $(\mathrm{C} 2)$. By definition, it is natural to compute the energy $\mathscr{E}$ defined in (2.4) for $\Psi^{\varepsilon}$. We can use (2.6) as in the proof of Lemma 2.1 to obtain

$$
0 \leq \mathscr{E}\left(\Psi^{\varepsilon}(0, x)\right)-\mathscr{E}(R)=O(\sqrt{\gamma})
$$

where $O(\cdot)$ depends only on $R, x_{0}, \xi_{0}$, and we used the fact that $R$ is the point of minimum for $\mathscr{E}$ on the manifold of functions with $L^{2}$ norm equal to $\sqrt{m}$. Moreover, we have the following

LEMma 3.1. There exist $\gamma_{0}>0$ such that for any $\varepsilon>0$ and any $\gamma \in\left(0, \gamma_{0}\right)$, there exists a positive constant depending only on $R, x_{0}$ and $\xi_{0}$ such that

$$
\begin{aligned}
& 0 \leq \mathscr{E}\left(\Psi^{\varepsilon}(t, x)\right)-\mathscr{E}(R) \\
& \leq m|\xi(t)|^{2}-\xi(t) \cdot \int_{\mathbb{R}^{N}} p_{\varepsilon}(t, x)+m V(x(t)) \\
& -\frac{1}{\varepsilon^{N}} \int_{\mathbb{R}^{N}} V(x)\left|u_{\varepsilon}(t, x)\right|^{2}+\operatorname{const}\left(R, x_{0}, \xi_{0}\right)\left(\sqrt{\gamma}+\varepsilon^{2}\right) \phi(\delta), \quad t>0 .
\end{aligned}
$$


Proof. The left inequality follows from the properties of $R$ and $\left\|\Psi^{\varepsilon}(t, \cdot)\right\|_{L^{2}}^{2}=$ $m$, for every $t>0$. Concerning the estimate from above, we use (2.9)-(2.10) to write

$$
\begin{aligned}
\mathscr{E}\left(\Psi^{\varepsilon}(t, x)\right) & =\frac{1}{2} \int_{\mathbb{R}^{N}}\left|\nabla \Psi^{\varepsilon}(t, x)\right|^{2}-\frac{1}{p+1} \int_{\mathbb{R}^{N}}\left|\Psi^{\varepsilon}(t, x)\right|^{2 p+2} \\
& =\left.\frac{1}{2} \int_{\mathbb{R}^{N}}|\nabla| u_{\varepsilon}(t, x(t)+\varepsilon x)\right|^{2} \\
& +\frac{1}{2} \int_{\mathbb{R}^{N}}\left|u_{\varepsilon}(t, x(t)+\varepsilon x)\right|^{2}\left|\xi(t)-\nabla\left(S_{\varepsilon}(t, x(t)+\varepsilon x)\right)\right|^{2} \\
& -\frac{1}{p+1} \int_{\mathbb{R}^{N}}\left|u_{\varepsilon}(t, x(t)+\varepsilon x)\right|^{2 p+2} \\
& =J_{\varepsilon}\left(u_{\varepsilon}, t\right)+K_{\varepsilon}\left(u_{\varepsilon}, t\right)-\frac{1}{\varepsilon^{N}} \int_{\mathbb{R}^{N}} V(x)\left|u_{\varepsilon}(t, x)\right|^{2} \\
& +\frac{1}{2} m|\xi(t)|^{2}-\int_{\mathbb{R}^{N}} p_{\varepsilon}(t, x) \cdot \xi(t), \quad t>0,
\end{aligned}
$$

where we have used the expressions (2.11)-(2.12) for the internal and kinetic energy of $u_{\varepsilon}$. Hence, we get

$$
\begin{aligned}
\mathscr{E}\left(\Psi^{\varepsilon}(t, x)\right)-\mathscr{E}(R) & =E_{\varepsilon}\left(u_{\varepsilon}, t\right)-\mathscr{E}(R)-m \mathcal{H}(x(t), \xi(t)) \\
& +m|\xi(t)|^{2}-\xi(t) \cdot \int_{\mathbb{R}^{N}} p_{\varepsilon}(t, x)+m V(x(t)) \\
& -\frac{1}{\varepsilon^{N}} \int_{\mathbb{R}^{N}} V(x)\left|u_{\varepsilon}(t, x)\right|^{2}, \quad t>0 .
\end{aligned}
$$

The assertion then follows from inequality (2.13) in Proposition 2.5.

Let us now introduce, for any $t>0$, the terms

$$
\eta_{1}^{\varepsilon}(t):=m \xi(t)-\int_{\mathbb{R}^{N}} p_{\varepsilon}(t, x), \quad \eta_{2}^{\varepsilon}(t):=m V(x(t))-\frac{1}{\varepsilon^{N}} \int_{\mathbb{R}^{N}} V(x)\left|u_{\varepsilon}(t, x)\right|^{2} .
$$

From Lemma 3.1 we have

$$
0 \leq \mathscr{E}\left(\Psi^{\varepsilon}(t, x)\right)-\mathscr{E}(R) \leq|\xi(t)|\left|\eta_{1}^{\varepsilon}(t)\right|+\left|\eta_{2}^{\varepsilon}(t)\right|+\operatorname{const}\left(R, x_{0}, \xi_{0}, \delta\right)\left(\sqrt{\gamma}+\varepsilon^{2}\right),
$$

for every $\varepsilon>0$ and $\gamma \in\left(0, \gamma_{0}\right)$. If we write, as in the proof of Lemma 3.1,

$$
\mathscr{E}\left(\Psi^{\varepsilon}(t, x)\right)=\mathscr{E}\left(\left|\Psi^{\varepsilon}(t, x)\right|\right)+\frac{1}{2 \varepsilon^{N}} \int_{\mathbb{R}^{N}}\left|u_{\varepsilon}(t, x)\right|^{2}\left|\xi(t)-\varepsilon \nabla S_{\varepsilon}(t, x)\right|^{2},
$$

from (3.3) and Lemma 3.1 we obtain

$$
\begin{aligned}
& \frac{1}{\varepsilon^{N}} \int_{\mathbb{R}^{N}}\left|v_{\varepsilon}(x)\right|^{2}\left|\xi_{0}-\varepsilon \nabla S_{\varepsilon}(0, x)\right|^{2}=O(\sqrt{\gamma}), \\
& \frac{1}{\varepsilon^{N}} \int_{\mathbb{R}^{N}}\left|u_{\varepsilon}(t, x)\right|^{2}\left|\xi(t)-\varepsilon \nabla S_{\varepsilon}(t, x)\right|^{2} \leq|\xi(t)|\left|\eta_{1}^{\varepsilon}(t)\right|+\left|\eta_{2}^{\varepsilon}(t)\right| \\
& +O\left(\sqrt{\gamma}+\varepsilon^{2}\right), \quad t>0,
\end{aligned}
$$

since $\mathscr{E}\left(\left|\Psi^{\varepsilon}(t, x)\right|\right)-\mathscr{E}(R) \geq 0$, for all $t \geq 0$.

Let us now recall the well-known quantitative property which follows from M. Weinstein modulational stability theory $[\mathbf{2 1}, \mathbf{2 2}]$. 
Proposition 3.2. There exist two positive constants $\mathcal{C}$ and $\mathcal{A}$ such that

$$
\inf _{\substack{\xi \in \mathbb{R}^{N} \\ \theta \in[0,2 \pi)}}\left\|\Psi-e^{\imath \theta} R(\cdot-\xi)\right\|_{H^{1}}^{2} \leq \mathcal{C}(\mathscr{E}(\Psi)-\mathscr{E}(R)),
$$

for every $\Psi \in H^{1}\left(\mathbb{R}^{N}\right)$ such that $\|\Psi\|_{L^{2}}=\|R\|_{L^{2}}$ and $\mathscr{E}(\Psi)-\mathscr{E}(R)<\mathcal{A}$.

Let us now fix a time $T>0, \varepsilon_{0}>0$ as in (3.15) and $\gamma_{0}>0$ as in Lemma 3.1. Let us set

$$
T^{\varepsilon, \gamma}:=\sup \left\{t \in[0, T]:|\xi(s)|\left|\eta_{1}^{\varepsilon}(s)\right|+\left|\eta_{2}^{\varepsilon}(s)\right| \leq \mu, \quad \text { for all } s \in(0, t)\right\},
$$

where $\mu>0$ is such that

$$
\mu+\operatorname{const}\left(R, x_{0}, \xi_{0}, \delta\right)\left(\sqrt{\gamma}+\varepsilon^{2}\right)<\mathcal{A}, \quad \text { for all } \varepsilon<\varepsilon_{0} \text { and } \gamma<\gamma_{0},
$$

where $\operatorname{const}\left(R, x_{0}, \xi_{0}, \delta\right)$ is as in (3.5) and $\mathcal{A}$ is as in Proposition 3.2, so that $\mathscr{E}\left(\Psi^{\varepsilon}(t, x)\right)-\mathscr{E}(R)<\mathcal{A}$ by virtue of $(3.5)$ for all $t \in\left[0, T^{\varepsilon, \gamma}\right)$. Then, in turn, Proposition 3.2 yields functions $\varpi^{\varepsilon}:\left[0, T^{\varepsilon, \gamma}\right) \rightarrow[0,2 \pi)$ and $w^{\varepsilon}:\left[0, T^{\varepsilon, \gamma}\right) \rightarrow \mathbb{R}^{N}$ such that

$$
\begin{aligned}
& \left\|\Psi^{\varepsilon}(t, x)-e^{\imath \varpi^{\varepsilon}(t)} R\left(x+w^{\varepsilon}(t)\right)\right\|_{H^{1}}^{2} \\
& \leq \mathcal{C}\left(|\xi(t)|\left|\eta_{1}^{\varepsilon}(t)\right|+\left|\eta_{2}^{\varepsilon}(t)\right|+\operatorname{const}\left(R, x_{0}, \xi_{0}, \delta\right)\left(\sqrt{\gamma}+\varepsilon^{2}\right)\right),
\end{aligned}
$$

for all $t \in\left[0, T^{\varepsilon, \gamma}\right)$. Then, we get the following

LEMMA 3.3. There exist families of functions $\theta^{\varepsilon}:\left[0, T^{\varepsilon, \gamma}\right) \rightarrow[0,2 \pi)$ and $x^{\varepsilon}$ : $\left[0, T^{\varepsilon, \gamma}\right) \rightarrow \mathbb{R}^{N}$ such that

$$
\begin{aligned}
& \left\|u_{\varepsilon}(t, x)-e^{\frac{\imath}{\varepsilon}\left(\xi(t) \cdot x+\theta^{\varepsilon}(t)\right)} R\left(\frac{x-x^{\varepsilon}(t)}{\varepsilon}\right)\right\|_{H_{\varepsilon}^{1}}^{2} \\
& \leq \mathcal{C}\left(|\xi(t)|\left|\eta_{1}^{\varepsilon}(t)\right|+\left|\eta_{2}^{\varepsilon}(t)\right|+\operatorname{const}\left(R, x_{0}, \xi_{0}\right)\left(\sqrt{\gamma}+\varepsilon^{2}\right)\right)
\end{aligned}
$$

for all $t \in\left[0, T^{\varepsilon, \gamma}\right)$.

Proof. In light of inequality (3.9), defining the functions $\theta^{\varepsilon}:\left[0, T^{\varepsilon, \gamma}\right) \rightarrow$ $[0,2 \pi)$ and $x^{\varepsilon}:\left[0, T^{\varepsilon, \gamma}\right) \rightarrow \mathbb{R}^{N}$ by setting $\theta^{\varepsilon}(t):=\varepsilon \varpi^{\varepsilon}(t)$ and $x^{\varepsilon}(t):=x(t)-\varepsilon w^{\varepsilon}(t)$ for every $\left[0, T^{\varepsilon, \gamma}\right)$ respectively, the assertion follows by the definition of $\Psi^{\varepsilon}$.

We now consider the behavior of the difference $\left|x^{\varepsilon}(t)-x(t)\right|$. This can be done as in [14], since the proofs do not depend on the properties of the potential $V$. Let $\chi$ denote the cuff-off function which is defined in $[\mathbf{1 4}, \mathrm{p.179]}$. Then we can get

LEMma 3.4. For every $t \in\left[0, T^{\varepsilon, \gamma}\right)$ we have

$$
\begin{aligned}
& \varepsilon\left|w^{\varepsilon}(t)\right|=\left|x^{\varepsilon}(t)-x(t)\right| \leq \operatorname{const}\left(R, x_{0}, \xi_{0}, \delta\right)\left(\left|\eta_{1}^{\varepsilon}(t)\right|\right. \\
& \left.+\left|\eta_{2}^{\varepsilon}(t)\right|+\left|\eta_{3}^{\varepsilon}(t)\right|+\sqrt{\gamma}+\varepsilon^{2}\right),
\end{aligned}
$$

where $\eta_{3}^{\varepsilon}(t)$ is defined as $\eta_{3}^{\varepsilon}(t):=\frac{1}{\varepsilon^{N}} \int_{\mathbb{R}^{N}} x \chi(x)\left|u_{\varepsilon}(t, x)\right|^{2}-m x(t)$ and it satisfies

$$
\begin{aligned}
& \eta_{3}^{\varepsilon}(0) \leq \operatorname{const}\left(R, x_{0}, \xi_{0}, \delta\right) \varepsilon^{2}, \\
& \left|\frac{d}{d t} \eta_{3}^{\varepsilon}(t)\right| \leq \operatorname{const}\left(R, x_{0}, \xi_{0}, \delta\right)\left(\left|\eta_{1}^{\varepsilon}(t)\right|\right. \\
& \left.+\left|\eta_{2}^{\varepsilon}(t)\right|+\left|\eta_{3}^{\varepsilon}(t)\right|+\sqrt{\gamma}+\varepsilon^{2}\right) .
\end{aligned}
$$


Proof. The proof of (3.10) follows by just mimicking step by step the proof of [14, Lemma 3.5], which is based on the arguments of [14, Lemma 3.4] in view of our inequalities (3.5)-(3.9). Notice also that in this proof one needs to choose the time $T$ properly, but depending only on $x_{0}, \xi_{0}, \varepsilon_{0}, \gamma_{0}$ and $\mathcal{A}$. This is analogous to $[\mathbf{1 4}$, Lemma 3.4]. Instead, concerning properties (3.11) it is sufficient to argue as in $[\mathbf{1 4}$, Lemma 3.6].

We now redefine the time $T^{\varepsilon, \gamma}$ by also imposing $w^{\varepsilon}$ to be bounded. Namely

$$
\begin{aligned}
T^{\varepsilon, \gamma}:= & \sup \left\{t \in[0, T]:|\xi(s)|\left|\eta_{1}^{\varepsilon}(s)\right|+\left|\eta_{2}^{\varepsilon}(s)\right| \leq \mu,\right. \\
& \text { and } \left.\left|w^{\varepsilon}(s)\right| \leq 1, \quad \text { for all } s \in(0, t)\right\}
\end{aligned}
$$

The last ingredients for the proof of the main result are estimates for the behavior of the quantities $\eta_{1}^{\varepsilon}$ and $\eta_{2}^{\varepsilon}$ defined in (3.4) in the interval $\left[0, T^{\varepsilon, \gamma}\right)$. It follows that these quantities have time derivatives bounded by

$$
\left|\eta^{\varepsilon}(t)\right|:=\left|\eta_{1}^{\varepsilon}(t)\right|+\left|\eta_{2}^{\varepsilon}(t)\right|+\left|\eta_{3}^{\varepsilon}(t)\right|
$$

up to an error depending on the kinetic energy $K_{\varepsilon}\left(u_{\varepsilon}, t\right)$ and on terms of the order $\sqrt{\gamma}+\varepsilon^{2}$

LEMmA 3.5. There exists positive constants only depending on $R, x_{0}$ and $\xi_{0}$ such that

$$
\left|\eta_{1}^{\varepsilon}(0)\right| \leq \operatorname{const}\left(R, x_{0}, \xi_{0}\right) \gamma^{\frac{1}{4}}, \quad\left|\eta_{2}^{\varepsilon}(0)\right| \leq \operatorname{const}\left(R, x_{0}, \xi_{0}\right)\left(\gamma+\varepsilon^{2}\right) .
$$

PRoOF. Let us recall the radial notation (2.10) for the momentum density. Then, we write

$$
\begin{aligned}
\left|\eta_{1}^{\varepsilon}(0)\right| & =\left|m \xi_{0}-\int_{\mathbb{R}^{N}} p_{\varepsilon}(0, x)\right|=\mid \frac{1}{\varepsilon^{N-1}} \int_{\mathbb{R}^{N}} R^{2}\left(\frac{x-x_{0}}{\varepsilon}\right) \frac{\xi_{0}}{\varepsilon} \\
& -\frac{1}{\varepsilon^{N-1}} \int_{\mathbb{R}^{N}}\left|v_{\varepsilon}(x)\right|^{2} \nabla S_{\varepsilon}(0, x) \mid \\
& =\mid \frac{1}{\varepsilon^{N-1}} \int_{\mathbb{R}^{N}} \frac{\xi_{0}}{\varepsilon}\left(R^{2}\left(\frac{x-x_{0}}{\varepsilon}\right)-\left|v_{\varepsilon}(x)\right|^{2}\right) \\
& +\frac{1}{\varepsilon^{N}} \int_{\mathbb{R}^{N}}\left|v_{\varepsilon}(x)\right|^{2}\left(\xi_{0}-\varepsilon \nabla S_{\varepsilon}(0, x)\right) \mid \\
& \leq\left.\left|\xi_{0}\right| \frac{1}{\varepsilon^{N}} \int_{\mathbb{R}^{N}}\left|R^{2}\left(\frac{x-x_{0}}{\varepsilon}\right)-\right| v_{\varepsilon}(x)\right|^{2} \mid \\
& +\left(\frac{1}{\varepsilon^{N}} \int_{\mathbb{R}^{N}}\left|v_{\varepsilon}(x)\right|^{2}\right)^{\frac{1}{2}}\left(\frac{1}{\varepsilon^{N}} \int_{\mathbb{R}^{N}}\left|v_{\varepsilon}(x)\right|^{2}\left|\xi_{0}-\varepsilon \nabla S_{\varepsilon}(0, x)\right|^{2}\right)^{\frac{1}{2}} \\
& \leq 2\left|\xi_{0}\right| \sqrt{m \gamma}+\operatorname{const}\left(R, x_{0}, \xi_{0}\right) \sqrt{m} \gamma^{\frac{1}{4}} \leq \operatorname{const}\left(R, x_{0}, \xi_{0}\right) \gamma^{\frac{1}{4}}
\end{aligned}
$$

where in the last line we have used the inequality for all $a, b \in \mathbb{C}$

$$
\left.\int|| a\right|^{2}-|b|^{2} \mid \leq\left(\int(|a|+|b|)^{2}\right)^{\frac{1}{2}}\left(\int|a-b|^{2}\right)^{\frac{1}{2}},
$$

condition $(\mathrm{C} 2)$ on $v_{\varepsilon}(x)$ and the estimate (3.6). The term $\eta_{2}^{\varepsilon}(0)$ is estimated in Lemma 2.2.

Let us now consider the increase rate in time. We can state the following 
Proposition 3.6. For every $t \in\left[0, T^{\varepsilon, \gamma}\right)$, we have

$$
\left|\frac{d}{d t} \eta_{1}^{\varepsilon}(t)\right| \leq \operatorname{const}\left(V, R, x_{0}, \xi_{0}, v_{\varepsilon}\right)\left(\left|\eta^{\varepsilon}(t)\right|+\sqrt{\gamma}+\varepsilon^{2}+T \frac{\left(K_{\varepsilon}\left(u_{\varepsilon}, t\right)-m V_{0}\right)^{\frac{1}{4}}}{\varepsilon^{\frac{1}{2}+3 \frac{2+\beta}{1-\beta}}}\right),
$$

for every \& small enough.

Proof. Let $\theta^{\varepsilon}$ be the family of functions introduced in Lemma 3.3. Then, using (1.6) and (2.2), we have

$$
\begin{aligned}
\left|\frac{d}{d t} \eta_{1}^{\varepsilon}(t)\right| & =\left.\left|m \nabla V(x(t))-\frac{1}{\varepsilon^{N}} \int_{\mathbb{R}^{N}} \nabla V(x)\right| u_{\varepsilon}(t, x)\right|^{2} \mid \\
& =\left.\left|\frac{1}{\varepsilon^{N}} \int_{\mathbb{R}^{N}}\right| u_{\varepsilon}(t, x)\right|^{2}[\nabla V(x(t))-\nabla V(x)] \mid \leq \\
& \leq\left|\frac{1}{\varepsilon^{N}} \int_{\mathbb{R}^{N}}\left(\left|u_{\varepsilon}(t, x)\right|-R\left(\frac{x-x^{\varepsilon}(t)}{\varepsilon}\right)\right)^{2}[\nabla V(x(t))-\nabla V(x)]\right|+ \\
& +\left|\frac{1}{\varepsilon^{N}} \int_{\mathbb{R}^{N}} R^{2}\left(\frac{x-x^{\varepsilon}(t)}{\varepsilon}\right)[\nabla V(x(t))-\nabla V(x)]\right|+ \\
& +\mid \frac{2}{\varepsilon^{N}} \int_{\mathbb{R}^{N}}\left(\left|u_{\varepsilon}(t, x)\right|-R\left(\frac{x-x^{\varepsilon}(t)}{\varepsilon}\right)\right) \\
& R\left(\frac{x-x^{\varepsilon}(t)}{\varepsilon}\right)[\nabla V(x(t))-\nabla V(x)] \mid= \\
& =: I_{1}+I_{2}+I_{3}
\end{aligned}
$$

where we used the elementary identity $|a|^{2}=(|a|-|b|)^{2}+|b|^{2}+2(|a|-|b|)|b|$. Let us estimate these terms, beginning with $I_{1}$.

$$
\begin{gathered}
I_{1}=\left|\frac{1}{\varepsilon^{N}} \int_{\mathbb{R}^{N}}\left(\left|u_{\varepsilon}(t, x)\right|-R\left(\frac{x-x^{\varepsilon}(t)}{\varepsilon}\right)\right)^{2}[\nabla V(x(t))-\nabla V(x)]\right| \leq \\
\leq \frac{1}{\varepsilon^{N}} \int_{\mathbb{R}^{N}}\left|u_{\varepsilon}(t, x)-R\left(\frac{x-x^{\varepsilon}(t)}{\varepsilon}\right) e^{\frac{\imath}{\varepsilon}\left(\xi(t) \cdot x+\theta^{\varepsilon}(t)\right)}\right|^{2}|\nabla V(x(t))-\nabla V(x)|
\end{gathered}
$$

Let $\tilde{\delta}=\delta\left(x_{0}, \xi_{0}\right) / 2$ so that $\operatorname{supp} v_{\varepsilon} \cap B(0, \tilde{\delta})=\emptyset$ by assumption (C3), and introduce a cut-off function $\psi_{\tilde{\delta}} \in C_{0}^{\infty}\left(\mathbb{R}^{N}\right)$ such that

$$
\psi_{\tilde{\delta}}(x)=\left\{\begin{array}{ll}
1, & |x| \leq \tilde{\delta}, \\
0, & |x| \geq \tilde{\tilde{\delta}},
\end{array} \quad\left|\nabla \psi_{\tilde{\delta}}(x)\right| \leq \frac{4}{\tilde{\delta}}, \quad \text { for } \quad \frac{\tilde{\delta}}{2} \leq|x| \leq \tilde{\delta}\right.
$$


Then we can write

$$
\begin{aligned}
I_{1} & \leq \frac{2}{\varepsilon^{N}} \int_{\mathbb{R}^{N}} \mid u_{\varepsilon}(t, x)-R\left(\frac{x-x^{\varepsilon}(t)}{\varepsilon}\right) e^{\left.\frac{\imath}{\varepsilon}\left(\xi(t) \cdot x+\theta^{\varepsilon}(t)\right)\right|^{2}} \\
& |\nabla V(x(t))-\nabla V(x)| \psi_{\tilde{\delta}}(x) \\
& +\frac{2}{\varepsilon^{N}} \int_{\mathbb{R}^{N}}\left|u_{\varepsilon}(t, x)-R\left(\frac{x-x^{\varepsilon}(t)}{\varepsilon}\right) e^{\frac{\imath}{\varepsilon}\left(\xi(t) \cdot x+\theta^{\varepsilon}(t)\right)}\right|^{2} \\
& |\nabla V(x(t))-\nabla V(x)|\left(1-\psi_{\tilde{\delta}}(x)\right) \\
& \leq \frac{2}{\varepsilon^{N}} \int_{B(0, \tilde{\delta})}\left|u_{\varepsilon}(t, x)-R\left(\frac{x-x^{\varepsilon}(t)}{\varepsilon}\right) e^{\frac{\imath}{\varepsilon}\left(\xi(t) \cdot x+\theta^{\varepsilon}(t)\right)}\right|^{2} \\
& (|\nabla V(x(t))|+|\nabla V(x)|) \psi_{\tilde{\delta}}(x) \\
& +\frac{4 \phi(\tilde{\delta} / 2)}{\varepsilon^{N}} \int_{\mathbb{R}^{N} \backslash B(0, \tilde{\delta} / 2)}\left|u_{\varepsilon}(t, x)-R\left(\frac{x-x^{\varepsilon}(t)}{\varepsilon}\right) e^{\frac{\imath}{\varepsilon}\left(\xi(t) \cdot x+\theta^{\varepsilon}(t)\right)}\right|^{2}
\end{aligned}
$$

where $\phi$ is defined in (1.2). By Lemma 3.3, inequality $|a-b|^{2} \leq 2|a|^{2}+2|b|^{2}$ and $\inf _{t \geq 0}|x(t)| \geq \delta$, we write

$$
\begin{aligned}
I_{1} & \leq \frac{4}{\varepsilon^{N}} \int_{B(0, \tilde{\delta})}\left|u_{\varepsilon}(t, x)\right|^{2}|\nabla V(x)| \psi_{\tilde{\delta}}(x)+\frac{4}{\varepsilon^{N}} \int_{B(0, \tilde{\delta})}\left|R\left(\frac{x-x^{\varepsilon}(t)}{\varepsilon}\right)\right|^{2}|\nabla V(x)| \\
& +\frac{2}{\varepsilon^{N}}(\phi(\delta)+2 \phi(\tilde{\delta} / 2)) \int_{\mathbb{R}^{N}} \mid u_{\varepsilon}(t, x)-R\left(\frac{x-x^{\varepsilon}(t)}{\varepsilon}\right) e^{\left.\frac{\imath}{\varepsilon}\left(\xi(t) \cdot x+\theta^{\varepsilon}(t)\right)\right|^{2}} \\
& \leq \frac{4}{\varepsilon^{N}} \int_{B(0, \tilde{\delta})}\left|u_{\varepsilon}(t, x)\right|^{2}|\nabla V(x)| \psi_{\tilde{\delta}}(x)+\frac{4}{\varepsilon^{N}} \int_{B(0, \tilde{\delta})}\left|R\left(\frac{x-x^{\varepsilon}(t)}{\varepsilon}\right)\right|^{2}|\nabla V(x)| \\
& +6 \max \{\phi(\delta), \phi(\tilde{\delta} / 2)\} \mathcal{C}\left(|\xi(t)|\left|\eta_{1}^{\varepsilon}(t)\right|+\left|\eta_{2}^{\varepsilon}(t)\right|+\operatorname{const}\left(R, x_{0}, \xi_{0}\right)\left(\sqrt{\gamma}+\varepsilon^{2}\right)\right) \\
& \leq \frac{4}{\varepsilon^{N}} \int_{B(0, \tilde{\delta})}\left|u_{\varepsilon}(t, x)\right|^{2}|\nabla V(x)| \psi_{\tilde{\delta}}(x)+4\|\nabla V\|_{L^{1}} \frac{1}{\varepsilon^{N}}\left\|R^{2}\right\| L_{L^{\infty}\left(\mathbb{R}^{N} \backslash B\left(0, \frac{\left|x^{\varepsilon}(t)\right|-\tilde{\delta}}{\varepsilon}\right)\right)} \\
& +6 \phi(\delta / 4) \mathcal{C}\left(|\xi(t)|\left|\eta_{1}^{\varepsilon}(t)\right|+\left|\eta_{2}^{\varepsilon}(t)\right|+\operatorname{const}\left(R, x_{0}, \xi_{0}\right)\left(\sqrt{\gamma}+\varepsilon^{2}\right),\right.
\end{aligned}
$$

and by (1.5) it holds for $\varepsilon$ small enough

$$
\left\|R^{2}\right\|_{L^{\infty}\left(\mathbb{R}^{N} \backslash B\left(0, \frac{\left|x^{\varepsilon}(t)\right|-\tilde{\delta}}{\varepsilon}\right)\right)} \leq \mathrm{const} \frac{\varepsilon^{N-1} e^{-\frac{\delta}{2 \varepsilon}}}{\delta^{N-1}},
$$

where, since $x^{\varepsilon}(t)=x(t)-\varepsilon w^{\varepsilon}(t),\left|w^{\varepsilon}(t)\right| \leq 1$ for $t \in\left[0, T^{\varepsilon, \gamma}\right)$ as defined in (3.12) and $|x(t)| \geq \delta$, for $\varepsilon$ small

$$
\frac{\left|x^{\varepsilon}(t)\right|-\tilde{\delta}}{\varepsilon} \geq \frac{|x(t)|-\varepsilon-\tilde{\delta}}{\varepsilon} \geq \frac{\delta-\tilde{\delta}-\varepsilon}{\varepsilon} \geq \frac{\delta}{4 \varepsilon} .
$$

Hence we choose $\varepsilon_{0}>0$ such that, for $\varepsilon<\varepsilon_{0}$

$$
\frac{1}{\varepsilon^{N}} \frac{\varepsilon^{N-1} e^{-\frac{\delta}{2 \varepsilon}}}{\delta^{N-1}}<\varepsilon^{2} .
$$

We obtain then

$$
\begin{aligned}
I_{1} \leq & \frac{4}{\varepsilon^{N}} \int_{B(0, \tilde{\delta})}\left|u_{\varepsilon}(t, x)\right|^{2}|\nabla V(x)| \psi_{\tilde{\delta}}(x)+\operatorname{const}\left(V, R, x_{0}, \xi_{0}\right) \\
& \mathcal{C}\left(|\xi(t)|\left|\eta_{1}^{\varepsilon}(t)\right|+\left|\eta_{2}^{\varepsilon}(t)\right|+\sqrt{\gamma}+\varepsilon^{2}\right) .
\end{aligned}
$$


We conclude the proof by showing that, for every $t \in\left[0, T^{\varepsilon, \gamma}\right)$, there holds

$$
\begin{aligned}
& \frac{1}{\varepsilon^{N}} \int_{B(0, \tilde{\delta})}\left|u_{\varepsilon}(t, x)\right|^{2}|\nabla V(x)| \psi_{\tilde{\delta}}(x) \\
& \leq \operatorname{const}\left(V, x_{0}, \xi_{0}, v_{\varepsilon}\right)\left(\varepsilon^{2}+T \frac{\left(K_{\varepsilon}\left(u_{\varepsilon}, t\right)-m V_{0}\right)^{\frac{1}{4}}}{\varepsilon^{\frac{1}{2}+3 \frac{2+\beta}{1-\beta}}}\right) .
\end{aligned}
$$

Let us introduce another cut-off at the origin, that is a function $\varphi_{\varepsilon} \in C_{0}^{\infty}\left(\mathbb{R}^{N} \backslash\{0\}\right)$, satisfying

$$
\varphi_{\varepsilon}(x)= \begin{cases}0, & |x| \leq r_{\varepsilon}^{\prime \prime}, \\ 1, & r_{\varepsilon}^{\prime} \leq|x| \leq 2 \tilde{\delta}, \quad\left|\nabla \varphi_{\varepsilon}(x)\right| \leq \frac{2}{r_{\varepsilon}^{\prime}-r_{\varepsilon}^{\prime \prime}}, \quad \text { for } \quad r_{\varepsilon}^{\prime \prime} \leq|x| \leq r_{\varepsilon}^{\prime}, \\ 0, & |x| \geq 3 \tilde{\delta},\end{cases}
$$

with $r_{\varepsilon}^{\prime}$ and $r_{\varepsilon}^{\prime \prime}$ to be chosen later, see formulas (3.24). By assumption (V1) and inequality (2.7) with the choice $q=2^{*}$, we apply Hölder inequality to obtain

$$
\begin{aligned}
& \frac{1}{\varepsilon^{N}} \int_{B(0, \tilde{\delta})}\left|u_{\varepsilon}(t, x)\right|^{2}|\nabla V(x)| \psi_{\tilde{\delta}}(x)\left(1-\varphi_{\varepsilon}(x)\right) \\
& \leq \frac{1}{\varepsilon^{N}} \int_{B\left(0, r_{\varepsilon}^{\prime}\right)}\left|u_{\varepsilon}(t, x)\right|^{2}|\nabla V(x)| \\
& \leq \operatorname{const}(N) \frac{1}{\varepsilon^{N}}\left\|\nabla u_{\varepsilon}(t, \cdot)\right\|_{L^{2}}^{2}\left(\int_{0}^{r_{\varepsilon}^{\prime}} \frac{1}{r^{\frac{N}{2}(\beta+1)}} r^{N-1} d r\right)^{\frac{2}{N}} \\
& \leq \operatorname{const}(N, \beta) M\left(x_{0}, \xi_{0}, v_{\varepsilon}\right) \frac{\left(r_{\varepsilon}^{\prime}\right)^{1-\beta}}{\varepsilon^{2}}
\end{aligned}
$$

where $M\left(x_{0}, \xi_{0}, v_{\varepsilon}\right)$ is defined in Lemma 2.3. We can then write

$$
\begin{aligned}
& \frac{1}{\varepsilon^{N}} \int_{B(0, \tilde{\delta})}\left|u_{\varepsilon}(t, x)\right|^{2}|\nabla V(x)| \psi_{\tilde{\delta}}(x) \\
& \leq \frac{1}{\varepsilon^{N}} \int_{B(0, \tilde{\delta})}\left|u_{\varepsilon}(t, x)\right|^{2}|\nabla V(x)| \psi_{\tilde{\delta}}(x) \varphi_{\varepsilon}(x)+\mathrm{const} \frac{\left(r_{\varepsilon}^{\prime}\right)^{1-\beta}}{\varepsilon^{2}}
\end{aligned}
$$

where the constant in the last term only depends on the initial conditions of (1.9). Moreover, by definition of $\tilde{\delta}$ and by virtue of identity (2.1), we have

$$
\int_{B(0, \tilde{\delta})}\left|u_{\varepsilon}(0, x)\right|^{2}|\nabla V(x)| \psi_{\tilde{\delta}}(x) \varphi_{\varepsilon}(x)=0
$$

Since $\psi_{\tilde{\delta}}(x) \varphi_{\varepsilon}(x) \in C_{0}^{\infty}(B(0, \tilde{\delta}) \backslash\{0\})$, there holds

$$
\begin{aligned}
& \frac{d}{d t}\left(\int_{B(0, \tilde{\delta})} \frac{\left|u_{\varepsilon}(t, x)\right|^{2}}{\varepsilon^{N}}|\nabla V(x)| \psi_{\tilde{\delta}}(x) \varphi_{\varepsilon}(x)\right) \\
& =\int_{B(0, \tilde{\delta})} p_{\varepsilon}(t, x) \cdot \nabla\left(|\nabla V(x)| \psi_{\tilde{\delta}}(x) \varphi_{\varepsilon}(x)\right),
\end{aligned}
$$

and to give an estimate for this last term we use the radial notation (2.10) for the momentum density and split the integral in three terms, where the properties of the cut-off functions $\psi_{\tilde{\delta}}$, see $(3.14)$, and $\varphi_{\varepsilon}$, see (3.18), are used to determine the 
domain of integration. We obtain

$$
\left|\int_{B(0, \tilde{\delta})} p_{\varepsilon}(t, x) \cdot \nabla\left(|\nabla V(x)| \psi_{\tilde{\delta}}(x) \varphi_{\varepsilon}(x)\right)\right| \leq J_{1}+J_{2}+J_{3},
$$

where we have set

$$
\begin{aligned}
& J_{1}:=\frac{1}{\varepsilon^{N}} \int_{B(0, \tilde{\delta}) \backslash B\left(0, r_{\varepsilon}^{\prime \prime}\right)}\left|u_{\varepsilon}(t, x)\right|^{2} \varepsilon\left|\nabla S_{\varepsilon}(t, x)\right||\nabla| \nabla V(x) \|, \\
& J_{2}:=\frac{1}{\varepsilon^{N}} \int_{B(0, \tilde{\delta}) \backslash B(0, \tilde{\delta} / 2)}\left|u_{\varepsilon}(t, x)\right|^{2} \varepsilon\left|\nabla S_{\varepsilon}(t, x)\right||\nabla V(x)|\left|\nabla \psi_{\tilde{\delta}}(x)\right|, \\
& J_{3}:=\frac{1}{\varepsilon^{N}} \int_{B\left(0, r_{\varepsilon}^{\prime}\right) \backslash B\left(0, r_{\varepsilon}^{\prime \prime}\right)}\left|u_{\varepsilon}(t, x)\right|^{2} \varepsilon\left|\nabla S_{\varepsilon}(t, x)\right||\nabla V(x)|\left|\nabla \varphi_{\varepsilon}(x)\right| .
\end{aligned}
$$

The estimates for the $J_{i}$ s are similar. We use Hölder inequality, assumptions (V1)(V3) and the estimate (2.14) for the kinetic energy $K_{\varepsilon}\left(u_{\varepsilon}, t\right)$ defined in (2.12). We obtain

$$
\begin{aligned}
& \varepsilon^{N} J_{1} \leq\left\|u_{\varepsilon}(t, \cdot)\right\|_{L^{2^{*}}}^{\frac{1}{2}}\left(\int\left|u_{\varepsilon}(t, x)\right|^{2} \varepsilon^{2}\left|\nabla S_{\varepsilon}(t, x)\right|^{2}\right)^{\frac{1}{2}} \\
& \left(\int\left|u_{\varepsilon}(t, x)\right|^{2}\left(V(x)-V_{0}\right)\right)^{\frac{1}{4}}\left(\int \frac{\left.|\nabla| \nabla V(x)\right|^{2 N}}{\left(V(x)-V_{0}\right)^{\frac{N}{2}}}\right)^{\frac{1}{2 N}},
\end{aligned}
$$

where all the integrals are computed on the set $B(0, \tilde{\delta}) \backslash B\left(0, r_{\varepsilon}^{\prime \prime}\right)$. Moreover we have

$$
\left\|u_{\varepsilon}(t, \cdot)\right\|_{L^{2^{*}}}^{\frac{1}{2}} \leq \operatorname{const}(N)\left\|\nabla u_{\varepsilon}(t, \cdot)\right\|_{L^{2}}^{\frac{1}{2}} \leq \operatorname{const}\left(N, x_{0}, \xi_{0}, v_{\varepsilon}\right) \varepsilon^{\frac{N-2}{4}},
$$

by the Gagliardo-Nirenberg inequality (2.7) and Lemma 2.3,

$$
\begin{gathered}
\left(\int_{B(0, \tilde{\delta}) \backslash B\left(0, r_{\varepsilon}^{\prime \prime}\right)}\left|u_{\varepsilon}(t, x)\right|^{2} \varepsilon^{2}\left|\nabla S_{\varepsilon}(t, x)\right|^{2}\right)^{\frac{1}{2}} \\
\leq\left(\int_{\mathbb{R}^{N}}\left|u_{\varepsilon}(t, x)\right|^{2} \varepsilon^{2}\left|\nabla S_{\varepsilon}(t, x)\right|^{2}\right)^{\frac{1}{2}} \leq\left(2 \varepsilon^{N} K_{\varepsilon}\left(u_{\varepsilon}, t\right)\right)^{\frac{1}{2}},
\end{gathered}
$$

by definition of $K_{\varepsilon}\left(u_{\varepsilon}, t\right)$ and the non-negativity of $V$,

$$
\begin{gathered}
\left(\int_{B(0, \tilde{\delta}) \backslash B\left(0, r_{\varepsilon}^{\prime \prime}\right)}\left|u_{\varepsilon}(t, x)\right|^{2}\left(V(x)-V_{0}\right)\right)^{\frac{1}{4}} \\
\leq\left(\int_{\mathbb{R}^{N}}\left|u_{\varepsilon}(t, x)\right|^{2}\left(V(x)-V_{0}\right)\right)^{\frac{1}{4}} \leq\left(\varepsilon^{N}\left(K_{\varepsilon}\left(u_{\varepsilon}, t\right)-m V_{0}\right)\right)^{\frac{1}{4}},
\end{gathered}
$$

by definition of $K_{\varepsilon}\left(u_{\varepsilon}, t\right)$ and the conservation of mass,

$$
\begin{aligned}
& \left(\int_{B(0, \tilde{\delta}) \backslash B\left(0, r_{\varepsilon}^{\prime \prime}\right)} \frac{\left.|\nabla| \nabla V(x)\right|^{2 N}}{\left(V(x)-V_{0}\right)^{\frac{N}{2}}}\right)^{\frac{1}{2 N}} \\
& \leq \operatorname{const}(N)\left(\int_{r_{\varepsilon}^{\prime \prime}}^{\tilde{\delta}} \frac{r^{-(\beta+2) 2 N}}{r^{-\beta \frac{N}{2}}} r^{N-1} d r\right)^{\frac{1}{2 N}}=\frac{\operatorname{const}(\delta, \beta, N)}{\left(r_{\varepsilon}^{\prime \prime}\right)^{\frac{3}{4}(2+\beta)}},
\end{aligned}
$$

by assumptions on the behavior of $V$ around the origin. Hence, putting the above facts together, we get

$$
J_{1} \leq \operatorname{const}\left(V, x_{0}, \xi_{0}, v_{\varepsilon}\right)\left(K_{\varepsilon}\left(u_{\varepsilon}, t\right)\right)^{\frac{1}{2}} \frac{\left(K_{\varepsilon}\left(u_{\varepsilon}, t\right)-m V_{0}\right)^{\frac{1}{4}}}{\left(r_{\varepsilon}^{\prime \prime}\right)^{\frac{3}{4}(2+\beta)} \varepsilon^{\frac{1}{2}}} .
$$


For the term $J_{2}$, we write

$$
\begin{gathered}
\varepsilon^{N} J_{2} \leq \frac{4}{\tilde{\delta}}\left(\int\left|u_{\varepsilon}(t, x)\right|^{2} \varepsilon^{2}\left|\nabla S_{\varepsilon}(t, x)\right|^{2}\right)^{\frac{1}{2}} \\
\left(\int\left|u_{\varepsilon}(t, x)\right|^{2}\left(V(x)-V_{0}\right)\right)^{\frac{1}{2}}\left\|\frac{|\nabla V(x)|}{\sqrt{V(x)-V_{0}}}\right\|_{L^{\infty}(B(0, \tilde{\delta}) \backslash B(0, \tilde{\delta} / 2))}
\end{gathered}
$$

where all the integrals are computed on $B(0, \tilde{\delta}) \backslash B(0, \tilde{\delta} / 2)$. For the first two integrals we proceed just as above. Concerning the third term, on account of conditions (V1) and (V3), we have

$$
\left\|\frac{|\nabla V(x)|}{\sqrt{V(x)-V_{0}}}\right\|_{L^{\infty}(B(0, \tilde{\delta}) \backslash B(0, \tilde{\delta} / 2))} \leq \frac{\phi(\tilde{\delta} / 2)}{\tilde{\delta}^{\frac{\beta}{2}}} .
$$

Hence, in turn, we can conclude

$$
J_{2} \leq \operatorname{const}\left(V, x_{0}, \xi_{0}, v_{\varepsilon}\right)\left(K_{\varepsilon}\left(u_{\varepsilon}, t\right)\right)^{\frac{1}{2}}\left(K_{\varepsilon}\left(u_{\varepsilon}, t\right)-m V_{0}\right)^{\frac{1}{2}} .
$$

Finally, concerning the term $J_{3}$, we write

$$
\begin{aligned}
& \varepsilon^{N} J_{3} \leq \frac{2\left\|u_{\varepsilon}(t, \cdot)\right\|_{L^{2^{*}}}^{\frac{1}{2}}}{r_{\varepsilon}^{\prime}-r_{\varepsilon}^{\prime \prime}}\left(\int\left|u_{\varepsilon}(t, x)\right|^{2} \varepsilon^{2}\left|\nabla S_{\varepsilon}(t, x)\right|^{2}\right)^{\frac{1}{2}} \\
& \left(\int\left|u_{\varepsilon}(t, x)\right|^{2}\left(V(x)-V_{0}\right)\right)^{\frac{1}{4}}\left(\int \frac{|\nabla V(x)|^{2 N}}{\left(V(x)-V_{0}\right)^{\frac{N}{2}}}\right)^{\frac{1}{2 N}},
\end{aligned}
$$

where all the integrals are computed on the set $B\left(0, r_{\varepsilon}^{\prime}\right) \backslash B\left(0, r_{\varepsilon}^{\prime \prime}\right)$. For the first three terms above we proceed as for $J_{1}$. Concerning the last term, we write

$$
\begin{aligned}
& \left(\int_{B\left(0, r_{\varepsilon}^{\prime}\right) \backslash B\left(0, r_{\varepsilon}^{\prime \prime}\right)} \frac{|\nabla V(x)|^{2 N}}{\left(V(x)-V_{0}\right)^{\frac{N}{2}}}\right)^{\frac{1}{2 N}} \\
& \leq \operatorname{const}(N)\left(\int_{r_{\varepsilon}^{\prime \prime}}^{r_{\varepsilon}^{\prime}} \frac{r^{-(\beta+1) 2 N}}{r^{-\beta \frac{N}{2}}} r^{N-1} d r\right)^{\frac{1}{2 N}} \\
& =\operatorname{const}(\delta, \beta, N)\left(\left(r_{\varepsilon}^{\prime \prime}\right)^{-N\left(1+\frac{3}{2} \beta\right)}-\left(r_{\varepsilon}^{\prime}\right)^{-N\left(1+\frac{3}{2} \beta\right)}\right)^{\frac{1}{2 N}} .
\end{aligned}
$$

Hence we finally get

$$
\begin{aligned}
& J_{3} \leq \operatorname{const}\left(V, x_{0}, \xi_{0}, v_{\varepsilon}\right)\left(K_{\varepsilon}\left(u_{\varepsilon}, t\right)\right)^{\frac{1}{2}}\left(K_{\varepsilon}\left(u_{\varepsilon}, t\right)-m V_{0}\right)^{\frac{1}{4}} \\
& \frac{\left(\left(r_{\varepsilon}^{\prime \prime}\right)^{-N\left(1+\frac{3}{2} \beta\right)}-\left(r_{\varepsilon}^{\prime}\right)^{-N\left(1+\frac{3}{2} \beta\right)}\right)^{\frac{1}{2 N}}}{\varepsilon^{\frac{1}{2}}\left(r_{\varepsilon}^{\prime}-r_{\varepsilon}^{\prime \prime}\right)} .
\end{aligned}
$$

The proof of the inequality (3.17) is finished by choosing

$$
r_{\varepsilon}^{\prime}=\varepsilon^{\frac{4}{1-\beta}}, \quad r_{\varepsilon}^{\prime \prime}=\frac{1}{2} r_{\varepsilon}^{\prime}
$$

taking (2.14) into account and using (3.19) and (3.20) together with (3.21), (3.22) and (3.23). This concludes the proof of the estimate of $I_{1}$.

Concerning the second term $I_{2}$, at first, take $\tilde{\delta}$ as above. Choosing $\varepsilon$ sufficiently small, as in (3.15), and in reasoning in a similar way, we have

$$
\frac{2}{\varepsilon^{N}} \int_{B(0, \tilde{\delta})} R^{2}\left(\frac{x-x^{\varepsilon}(t)}{\varepsilon}\right)|\nabla V(x(t))-\nabla V(x)| \leq \operatorname{const}(V, R) \varepsilon^{2} .
$$


So, we consider $\tilde{V} \in C^{2}\left(\mathbb{R}^{N}, \mathbb{R}\right)$ such that $\tilde{V}(x)=V(x)$ on $\mathbb{R}^{N} \backslash B(0, \tilde{\delta})$ and we get

$$
I_{2} \leq\left|\frac{2}{\varepsilon^{N}} \int_{\mathbb{R}^{N}} R^{2}\left(\frac{x-x^{\varepsilon}(t)}{\varepsilon}\right)[\nabla \tilde{V}(x(t))-\nabla \tilde{V}(x)]\right|+\operatorname{const}(V, R) \varepsilon^{2} .
$$

It holds

$$
\begin{aligned}
& \left|\int_{\mathbb{R}^{N}} R^{2}\left(\frac{x-x^{\varepsilon}(t)}{\varepsilon}\right)[\nabla \tilde{V}(x(t))-\nabla \tilde{V}(x)]\right| \leq \\
& \left|\int_{\mathbb{R}^{N}} R^{2}\left(\frac{x-x^{\varepsilon}(t)}{\varepsilon}\right)\left[\nabla \tilde{V}\left(x^{\varepsilon}(t)\right)-\nabla \tilde{V}(x)\right]\right|+ \\
& \left|\int_{\mathbb{R}^{N}} R^{2}\left(\frac{x-x^{\varepsilon}(t)}{\varepsilon}\right)\left[\nabla \tilde{V}(x(t))-\nabla \tilde{V}\left(x^{\varepsilon}(t)\right)\right]\right|
\end{aligned}
$$

And, in light of Lemma 3.4, it holds

$$
\begin{aligned}
& \left|\frac{2}{\varepsilon^{N}} \int_{\mathbb{R}^{N}} R^{2}\left(\frac{x-x^{\varepsilon}(t)}{\varepsilon}\right)\left[\nabla \tilde{V}(x(t))-\nabla \tilde{V}\left(x^{\varepsilon}(t)\right)\right]\right| \\
& \leq \operatorname{const}\left(R, x_{0}, \xi_{0}, \delta, V\right)\left(\left|\eta^{\varepsilon}(t)\right|+\sqrt{\gamma}+\varepsilon^{2}\right) .
\end{aligned}
$$

So we have that

$$
\begin{gathered}
I_{2} \leq\left|\frac{2}{\varepsilon^{N}} \int_{\mathbb{R}^{N}} R^{2}\left(\frac{x-x^{\varepsilon}(t)}{\varepsilon}\right)\left[\nabla \tilde{V}\left(x^{\varepsilon}(t)\right)-\nabla \tilde{V}(x)\right] d x\right|+ \\
\operatorname{const}\left(R, x_{0}, \xi_{0}, \delta, V\right)\left(\left|\eta^{\varepsilon}(t)\right|+\sqrt{\gamma}+\varepsilon^{2}\right) .
\end{gathered}
$$

Let us first write

$$
\begin{aligned}
& \frac{2}{\varepsilon^{N}} \int_{\mathbb{R}^{N}} R^{2}\left(\frac{x-x^{\varepsilon}(t)}{\varepsilon}\right)\left[\nabla \tilde{V}\left(x^{\varepsilon}(t)\right)-\nabla \tilde{V}(x)\right] d x \\
& =2 \int_{\mathbb{R}^{N}} R^{2}(y)\left[\nabla \tilde{V}\left(x^{\varepsilon}(t)\right)-\nabla \tilde{V}\left(x^{\varepsilon}(t)+\varepsilon y\right)\right] d y .
\end{aligned}
$$

By virtue of [14, Lemma 3.3] we conclude

$$
I_{2} \leq \operatorname{const}\left(R, x_{0}, \xi_{0}, \delta, V\right)\left(\left|\eta^{\varepsilon}(t)\right|+\sqrt{\gamma}+\varepsilon^{2}\right) .
$$

For $I_{3}$ we write

$$
\begin{gathered}
I_{3} \leq 2\left(\int_{\mathbb{R}^{N}} \frac{1}{\varepsilon^{N}}\left|u_{\varepsilon}(t, x)-R\left(\frac{x-x^{\varepsilon}(t)}{\varepsilon}\right)\right|^{2}\right)^{\frac{1}{2}} \\
\left(\int_{\mathbb{R}^{N}} \frac{1}{\varepsilon^{N}}|\nabla V(x(t))-\nabla V(x)|^{2} R^{2}\left(\frac{x-x^{\varepsilon}(t)}{\varepsilon}\right)\right)^{\frac{1}{2}} \leq \\
\leq \operatorname{const}\left(R, x_{0}, \xi_{0}\right)\left(\left|\eta_{1}^{\varepsilon}(t)\right|+\left|\eta_{2}^{\varepsilon}(t)\right|+\sqrt{\gamma}+\varepsilon^{2}\right)
\end{gathered}
$$

arguing as in the previous estimates.

This concludes the proof.

For $\varepsilon$ small, let us set

$$
\rho_{\varepsilon}^{\prime}=\varepsilon^{\frac{4}{2-\beta}}, \quad \rho_{\varepsilon}^{\prime \prime}=\frac{1}{2} \rho_{\varepsilon}^{\prime},
$$

introduce a cut-off function

$$
\chi_{\varepsilon}(x)=\left\{\begin{array}{ll}
0 & |x| \leq \rho_{\varepsilon}^{\prime \prime}, \\
1 & |x| \geq \rho_{\varepsilon}^{\prime},
\end{array} \quad\left|\nabla \chi_{\varepsilon}(x)\right| \leq \frac{2}{\rho_{\varepsilon}^{\prime}-\rho_{\varepsilon}^{\prime \prime}} \quad \text { for } \rho_{\varepsilon}^{\prime \prime} \leq|x| \leq \rho_{\varepsilon}^{\prime},\right.
$$


and, finally, define

$$
\tilde{\eta}_{2}^{\varepsilon}(t):=m V(x(t))-\frac{1}{\varepsilon^{N}} \int_{\mathbb{R}^{N}}\left|u_{\varepsilon}(t, x)\right|^{2} V(x) \chi_{\varepsilon}(x), \quad t \in\left[0, T^{\varepsilon, \gamma}\right) .
$$

Then, we have the following

Proposition 3.7. For every $t \in\left[0, T^{\varepsilon, \gamma}\right)$ we have

$$
\left|\eta_{2}^{\varepsilon}(t)\right| \leq\left|\tilde{\eta}_{2}^{\varepsilon}(t)\right|+\operatorname{const}\left(x_{0}, \xi_{0}, v_{\varepsilon}\right) \varepsilon^{2}
$$

with

$$
\left|\tilde{\eta}_{2}^{\varepsilon}(0)\right| \leq \operatorname{const}\left(R, x_{0}, \xi_{0}, v_{\varepsilon}\right)\left(\gamma+\varepsilon^{2}\right)
$$

and

$$
\begin{aligned}
& \left|\frac{d}{d t} \tilde{\eta}_{2}^{\varepsilon}(t)\right| \leq \operatorname{const}\left(V, R, x_{0}, \xi_{0}, v_{\varepsilon}\right)\left[\left|\eta^{\varepsilon}(t)\right|+\sqrt{\gamma}+\varepsilon^{2}\right. \\
& \left.\quad+\left(K_{\varepsilon}\left(u_{\varepsilon}, t\right)-m V_{0}\right)^{\frac{1}{4}}\left(\frac{1}{\varepsilon^{1+2 \frac{2+3 \beta}{2-\beta}}}+\frac{T}{\varepsilon^{\frac{1}{2}+3 \frac{2+\beta}{1-\beta}}}\right)\right] .
\end{aligned}
$$

PROOF. We first estimate the behavior of $\eta_{2}^{\varepsilon}$ near the origin. We can write

$$
\begin{aligned}
\eta_{2}^{\varepsilon}(t) & =m V(x(t))-\frac{1}{\varepsilon^{N}} \int_{\mathbb{R}^{N}}\left|u_{\varepsilon}(t, x)\right|^{2} V(x) \chi_{\varepsilon}(x) \\
& -\frac{1}{\varepsilon^{N}} \int_{\mathbb{R}^{N}}\left|u_{\varepsilon}(t, x)\right|^{2} V(x)\left(1-\chi_{\varepsilon}(x)\right)
\end{aligned}
$$

Moreover by Hölder inequality, inequality (2.7), Lemma 2.3 and assumption (V1),

$$
\begin{aligned}
& \left.\left|\frac{1}{\varepsilon^{N}} \int_{\mathbb{R}^{N}}\right| u_{\varepsilon}(t, x)\right|^{2} V(x)\left(1-\chi_{\varepsilon}(x)\right) \mid \\
& \leq \frac{1}{\varepsilon^{N}} \int_{B\left(0, \rho_{\varepsilon}^{\prime}\right)}\left|u_{\varepsilon}(t, x)\right|^{2} V(x) \\
& \leq \frac{1}{\varepsilon^{N}}\left\|u_{\varepsilon}\right\|_{L^{2^{*}}}^{2}\left(\int_{B\left(0, \rho_{\varepsilon}^{\prime}\right)} V(x)^{\frac{N}{2}}\right)^{\frac{2}{N}} \leq \operatorname{const}\left(x_{0}, \xi_{0}, v_{\varepsilon}\right) \frac{\left(\rho_{\varepsilon}^{\prime}\right)^{2-\beta}}{\varepsilon^{2}} .
\end{aligned}
$$

Whence, there holds

$$
\left|\eta_{2}^{\varepsilon}(t)\right| \leq\left|\tilde{\eta}_{2}^{\varepsilon}(t)\right|+\operatorname{const}\left(x_{0}, \xi_{0}, v_{\varepsilon}\right) \frac{\left(\rho_{\varepsilon}^{\prime}\right)^{2-\beta}}{\varepsilon^{2}}=\left|\tilde{\eta}_{2}^{\varepsilon}(t)\right|+\operatorname{const}\left(x_{0}, \xi_{0}, v_{\varepsilon}\right) \varepsilon^{2} .
$$

by (3.25). Using also Lemma 3.5 the estimate for $\left|\tilde{\eta}_{2}^{\varepsilon}(0)\right|$ follows.

Using formulas (1.6) and (2.1) and the radial notation (2.10) for the momentum density, we have

$$
\begin{aligned}
\left|\frac{d}{d t} \tilde{\eta}_{2}^{\varepsilon}(t)\right| & =\left|m \nabla V(x(t)) \cdot \xi(t)+\int_{\mathbb{R}^{N}}\left(\nabla \cdot p_{\varepsilon}(t, x)\right) V(x) \chi_{\varepsilon}(x)\right| \\
& \leq\left.\left|\frac{1}{\varepsilon^{N}} \int_{\mathbb{R}^{N}}\right| u_{\varepsilon}(t, x)\right|^{2}(\nabla V(x(t)) \cdot \xi(t)) \chi_{\varepsilon}(x) \\
& -\frac{1}{\varepsilon^{N}} \int_{\mathbb{R}^{N}}\left|u_{\varepsilon}(t, x)\right|^{2}\left(\varepsilon \nabla S_{\varepsilon}(t, x) \cdot \nabla V(x)\right) \chi_{\varepsilon}(x) \mid \\
& +\left.\left|\frac{1}{\varepsilon^{N}} \int_{\mathbb{R}^{N}}\right| u_{\varepsilon}(t, x)\right|^{2}(\nabla V(x(t)) \cdot \xi(t))\left(1-\chi_{\varepsilon}(x)\right) \mid \\
& +\left.\left|\frac{1}{\varepsilon^{N}} \int_{\mathbb{R}^{N}}\right| u_{\varepsilon}(t, x)\right|^{2}\left(\varepsilon \nabla S_{\varepsilon}(t, x) \cdot \nabla \chi_{\varepsilon}(x)\right) V(x) \mid=: I_{1}+I_{2}+I_{3} .
\end{aligned}
$$


Let us estimate these terms, beginning with $I_{1}$. By adding and subtracting

$$
\left|u_{\varepsilon}(t, x)\right|^{2} \nabla V(x) \cdot \xi(t),
$$

we write

$$
\begin{aligned}
I_{1} & \leq\left.\left|\frac{1}{\varepsilon^{N}} \int_{\mathbb{R}^{N}}\right| u_{\varepsilon}(t, x)\right|^{2}[\nabla V(x(t))-\nabla V(x)] \cdot \xi(t) \chi_{\varepsilon}(x) \mid \\
& +\left.\left|\frac{1}{\varepsilon^{N}} \int_{\mathbb{R}^{N}}\right| u_{\varepsilon}(t, x)\right|^{2} \nabla V(x) \cdot\left[\xi(t)-\varepsilon \nabla S_{\varepsilon}(t, x)\right] \chi_{\varepsilon}(x) \mid \\
& \leq \operatorname{const}\left(V, R, x_{0}, \xi_{0}, v_{\varepsilon}\right)\left(\left|\eta^{\varepsilon}(t)\right|+\sqrt{\gamma}+\varepsilon^{2}+T \frac{\left(K_{\varepsilon}\left(u_{\varepsilon}, t\right)-m V_{0}\right)^{\frac{1}{4}}}{\varepsilon^{\frac{1}{2}+3 \frac{2+\beta}{1-\beta}}}\right)+ \\
& +\left.\left|\frac{1}{\varepsilon^{N}} \int_{\mathbb{R}^{N}}\right| u_{\varepsilon}(t, x)\right|^{2} \nabla V(x) \cdot\left[\xi(t)-\varepsilon \nabla S_{\varepsilon}(t, x)\right] \chi_{\varepsilon}(x) \mid,
\end{aligned}
$$

where we have used the estimate of Proposition 3.6 for the derivative of $\eta_{1}^{\varepsilon}$. Moreover

$$
\begin{aligned}
& \left.\left|\frac{1}{\varepsilon^{N}} \int_{\mathbb{R}^{N}}\right| u_{\varepsilon}(t, x)\right|^{2} \nabla V(x) \cdot\left[\xi(t)-\varepsilon \nabla S_{\varepsilon}(t, x)\right] \chi_{\varepsilon}(x) \mid \\
& \leq \frac{1}{2 \varepsilon^{N}} \int_{\mathbb{R}^{N}}\left|u_{\varepsilon}(t, x)\right|^{2}\left|\xi(t)-\varepsilon \nabla S_{\varepsilon}(t, x)\right|^{2}+\frac{1}{2 \varepsilon^{N}} \int_{\mathbb{R}^{N}}\left|u_{\varepsilon}(t, x)\right|^{2}|\nabla V(x)|^{2} \chi_{\varepsilon}(x) \\
& \leq \frac{1}{2}|\xi(t)|\left|\eta_{1}^{\varepsilon}(t)\right| \\
& +\frac{1}{2}\left|\eta_{2}^{\varepsilon}(t)\right|+\operatorname{const}\left(R, x_{0}, \xi_{0}\right)\left(\sqrt{\gamma}+\varepsilon^{2}\right)+\frac{1}{2 \varepsilon^{N}} \int_{\mathbb{R}^{N}}\left|u_{\varepsilon}(t, x)\right|^{2}|\nabla V(x)|^{2} \chi_{\varepsilon}(x),
\end{aligned}
$$

by virtue of inequality (3.7). Finally, by Hölder inequality and the definition of $\chi_{\varepsilon}$ in (3.26), we get

$$
\begin{gathered}
\int_{\mathbb{R}^{N}}\left|u_{\varepsilon}(t, x)\right|^{2}|\nabla V(x)|^{2} \chi_{\varepsilon}(x) \leq\left\|u_{\varepsilon}(t, \cdot)\right\|_{L^{2^{*}}}\left(\int_{\mathbb{R}^{N}}\left|u_{\varepsilon}(t, x)\right|^{2}\left(V(x)-V_{0}\right)\right)^{\frac{1}{2}} \\
\left(\int_{\mathbb{R}^{N}} \frac{|\nabla V(x)|^{2 N}}{\left(V(x)-V_{0}\right)^{\frac{N}{2}}} \chi_{\varepsilon}^{N}(x)\right)^{\frac{1}{N}},
\end{gathered}
$$

and we can use the estimates

$$
\left\|u_{\varepsilon}(t, \cdot)\right\|_{L^{2^{*}}} \leq \operatorname{const}(N)\left\|\nabla u_{\varepsilon}(t \cdot)\right\|_{L^{2}} \leq \operatorname{const}\left(N, x_{0}, \xi_{0}, v_{\varepsilon}\right) \varepsilon^{\frac{N-2}{2}},
$$

via inequality (2.7) and Lemma 2.3,

$$
\left(\int_{\mathbb{R}^{N}}\left|u_{\varepsilon}(t, x)\right|^{2}\left(V(x)-V_{0}\right)\right)^{\frac{1}{2}} \leq\left(\varepsilon^{N}\left(K_{\varepsilon}\left(u_{\varepsilon}, t\right)-m V_{0}\right)\right)^{\frac{1}{2}}
$$

by definition of $K_{\varepsilon}\left(u_{\varepsilon}, t\right)$ and the conservation of mass,

$$
\begin{gathered}
\left(\int_{\mathbb{R}^{N}} \frac{|\nabla V(x)|^{2 N}}{\left(V(x)-V_{0}\right)^{\frac{N}{2}}} \chi_{\varepsilon}^{N}(x)\right)^{\frac{1}{N}} \leq \operatorname{const}(V, N)\left(\int_{\rho_{\varepsilon}^{\prime \prime}}^{1} \frac{r^{-(\beta+1) 2 N}}{r^{-\beta \frac{N}{2}}} r^{N-1} d r\right)^{\frac{1}{N}} \\
=\operatorname{const}(V, \delta, \beta)\left(\rho_{\varepsilon}^{\prime \prime}\right)^{-\left(1+\frac{3}{2} \beta\right)},
\end{gathered}
$$

by assumptions (V1) and (V2). Hence, we obtain

$$
\begin{aligned}
I_{1} \leq & \operatorname{const}\left(R, V, x_{0}, \xi_{0}\right)\left[\left|\eta_{1}^{\varepsilon}(t)\right|+\left|\eta_{2}^{\varepsilon}(t)\right|+\sqrt{\gamma}+\varepsilon^{2}\right. \\
& \left.+T \frac{\left(K_{\varepsilon}\left(u_{\varepsilon}, t\right)-m V_{0}\right)^{\frac{1}{4}}}{\varepsilon^{\frac{1}{2}+3 \frac{2+\beta}{1-\beta}}}+\frac{\left(K_{\varepsilon}\left(u_{\varepsilon}, t\right)-m V_{0}\right)^{\frac{1}{2}}}{\varepsilon\left(\rho_{\varepsilon}^{\prime \prime}\right)^{\left(1+\frac{3}{2} \beta\right)}}\right] .
\end{aligned}
$$


We now turn to the estimate for $I_{2}$. By assumption (V3), (1.7) and the definition of $\chi_{\varepsilon}$, we write

$$
I_{2} \leq \phi(\delta)|\xi(t)| \frac{1}{\varepsilon^{N}} \int_{B\left(0, \rho_{\varepsilon}^{\prime}\right)}\left|u_{\varepsilon}(t, x)\right|^{2},
$$

and by Hölder inequality, (2.7) and Lemma 2.3, we obtain

$$
\begin{aligned}
I_{2} \leq & \operatorname{const}\left(R, V, x_{0}, \xi_{0}\right)|\xi(t)| \varepsilon^{-N}\left\|u_{\varepsilon}\right\|_{L^{2^{*}}}^{2}\left(\rho_{\varepsilon}^{\prime}\right)^{2} \\
& \leq \operatorname{const}\left(R, V, x_{0}, \xi_{0}, v_{\varepsilon}\right)|\xi(t)| \frac{\left(\rho_{\varepsilon}^{\prime}\right)^{2}}{\varepsilon^{2}} .
\end{aligned}
$$

We now estimate $I_{3}$. We apply again Hölder inequality and the properties of $\chi_{\varepsilon}$ to get

$$
\begin{gathered}
\varepsilon^{N} I_{3} \leq \frac{2\left\|u_{\varepsilon}\right\|_{L^{2^{*}}}^{\frac{1}{2}}}{\rho_{\varepsilon}^{\prime}-\rho_{\varepsilon}^{\prime \prime}}\left(\int\left|u_{\varepsilon}(t, x)\right|^{2} \varepsilon^{2}\left|\nabla S_{\varepsilon}(t, x)\right|^{2}\right)^{\frac{1}{2}} \\
\left(\int\left|u_{\varepsilon}(t, x)\right|^{2}\left(V(x)-V_{0}\right)\right)^{\frac{1}{4}}\left(\int \frac{V(x)^{2 N}}{\left(V(x)-V_{0}\right)^{\frac{N}{2}}}\right)^{\frac{1}{2 N}},
\end{gathered}
$$

where all integrals are computed on $B\left(0, \rho_{\varepsilon}^{\prime}\right) \backslash B\left(0, \rho_{\varepsilon}^{\prime \prime}\right)$. Hence, we have

$$
\left\|u_{\varepsilon}(t, \cdot)\right\|_{L^{2^{*}}}^{\frac{1}{2}} \leq \operatorname{const}(N)\left\|\nabla u_{\varepsilon}(t, \cdot)\right\|_{L^{2}}^{\frac{1}{2}} \leq \operatorname{const}\left(N, x_{0}, \xi_{0}, v_{\varepsilon}\right) \varepsilon^{\frac{N-2}{4}}
$$

by inequality (2.7) and Lemma 2.3 ,

$$
\begin{gathered}
\left(\int_{B\left(0, \rho_{\varepsilon}^{\prime}\right) \backslash B\left(0, \rho_{\varepsilon}^{\prime \prime}\right)}\left|u_{\varepsilon}(t, x)\right|^{2} \varepsilon^{2}\left|\nabla S_{\varepsilon}(t, x)\right|^{2}\right)^{\frac{1}{2}} \\
\leq\left(\int_{\mathbb{R}^{N}}\left|u_{\varepsilon}(t, x)\right|^{2} \varepsilon^{2}\left|\nabla S_{\varepsilon}(t, x)\right|^{2}\right)^{\frac{1}{2}} \leq\left(\varepsilon^{N} K_{\varepsilon}\left(u_{\varepsilon}, t\right)\right)^{\frac{1}{2}}
\end{gathered}
$$

by definition of $K_{\varepsilon}\left(u_{\varepsilon}, t\right)$ and by the non-negativity of $V$,

$$
\begin{gathered}
\left(\int_{B\left(0, \rho_{\varepsilon}^{\prime}\right) \backslash B\left(0, \rho_{\varepsilon}^{\prime \prime}\right)}\left|u_{\varepsilon}(t, x)\right|^{2}\left(V(x)-V_{0}\right)\right)^{\frac{1}{4}} \leq\left(\int_{\mathbb{R}^{N}}\left|u_{\varepsilon}(t, x)\right|^{2}\left(V(x)-V_{0}\right)\right)^{\frac{1}{4}} \\
\leq\left(\varepsilon^{N}\left(K_{\varepsilon}\left(u_{\varepsilon}, t\right)-m V_{0}\right)\right)^{\frac{1}{4}},
\end{gathered}
$$

by definition of $K_{\varepsilon}\left(u_{\varepsilon}, t\right)$ and the conservation of mass,

$$
\begin{aligned}
& \left(\int_{B\left(0, \rho_{\varepsilon}^{\prime}\right) \backslash B\left(0, \rho_{\varepsilon}^{\prime \prime}\right)} \frac{V(x)^{2 N}}{\left(V(x)-V_{0}\right)^{\frac{N}{2}}}\right)^{\frac{1}{N}} \\
& \leq \operatorname{const}(V, N)\left(\int_{\rho_{\varepsilon}^{\prime \prime}}^{\rho_{\varepsilon}^{\prime}} r^{-\beta \frac{3}{2} N} r^{N-1} d r\right)^{\frac{1}{N}} \\
& =\operatorname{const}(\delta, \beta, N)\left|\left(\rho_{\varepsilon}^{\prime \prime}\right)^{N\left(1-\frac{3}{2} \beta\right)}-\left(\rho_{\varepsilon}^{\prime}\right)^{N\left(1-\frac{3}{2} \beta\right)}\right|^{\frac{1}{N}},
\end{aligned}
$$

by the assumptions (V1). Hence

$$
\begin{gathered}
I_{3} \leq \operatorname{const}\left(R, V, x_{0}, \xi_{0}\right)\left(K_{\varepsilon}\left(u_{\varepsilon}, t\right)\right)^{\frac{1}{2}}\left(K_{\varepsilon}\left(u_{\varepsilon}, t\right)-m V_{0}\right)^{\frac{1}{4}} \\
\frac{\left|\left(\rho_{\varepsilon}^{\prime \prime}\right)^{N\left(1-\frac{3}{2} \beta\right)}-\left(\rho_{\varepsilon}^{\prime}\right)^{N\left(1-\frac{3}{2} \beta\right)}\right|^{\frac{1}{N}}}{\varepsilon^{\frac{1}{2}}\left(\rho_{\varepsilon}^{\prime}-\rho_{\varepsilon}^{\prime \prime}\right)} .
\end{gathered}
$$

Taking into account (3.25) the assertion finally follows from inequalities (3.27), (3.28) and (3.29). 


\section{Proof of the main result completed}

Taking into account conditions (1.10) and inequality (2.14), we can find a const $\left(R, \xi_{0}\right)$ such that

$K_{\varepsilon}\left(u_{\varepsilon}, t\right)-m V_{0} \leq \frac{1}{2} m\left|\xi_{0}\right|^{2}+\frac{1}{\varepsilon^{N}} \int_{\mathbb{R}^{N}}\left(V(x)-V_{0}\right)\left|v_{\varepsilon}(x)\right|^{2}+\operatorname{const}\left(R, \xi_{0}\right) \sqrt{\gamma} \leq \varepsilon^{2 \frac{17+\beta}{1-\beta}}$.

Then, by Propositions 3.6-3.7, for all $t \in\left[0, T^{\varepsilon, \gamma}\right)$ we have

$$
\left|\eta_{2}^{\varepsilon}(t)\right| \leq \operatorname{const}\left(x_{0}, \xi_{0}, v_{\varepsilon}\right)\left(\left|\tilde{\eta}_{2}^{\varepsilon}(t)\right|+\varepsilon^{2}\right)
$$

and

$$
\begin{aligned}
\left|\frac{d}{d t} \eta_{1}^{\varepsilon}(t)\right| & \leq \operatorname{const}\left(V, R, x_{0}, \xi_{0}, v_{\varepsilon}\right)\left(\left|\eta_{1}^{\varepsilon}(t)\right|+\left|\tilde{\eta}_{2}^{\varepsilon}(t)\right|+\left|\eta_{3}^{\varepsilon}(t)\right|+\varepsilon^{2}\right) \\
\left|\frac{d}{d t} \tilde{\eta}_{2}^{\varepsilon}(t)\right| & \leq \operatorname{const}\left(V, R, x_{0}, \xi_{0}, v_{\varepsilon}\right)\left(\left|\eta_{1}^{\varepsilon}(t)\right|+\left|\tilde{\eta}_{2}^{\varepsilon}(t)\right|+\left|\eta_{3}^{\varepsilon}(t)\right|+\varepsilon^{2}\right)
\end{aligned}
$$

Furthermore, by virtue of Lemma 3.4, for every $t \in\left[0, T^{\varepsilon, \gamma}\right)$ we have

$$
\begin{aligned}
\left|\frac{d}{d t} \eta_{3}^{\varepsilon}(t)\right| & \leq \operatorname{const}\left(R, x_{0}, \xi_{0}, \delta\right)\left(\left|\eta_{1}^{\varepsilon}(t)\right|+\left|\eta_{2}^{\varepsilon}(t)\right|+\left|\eta_{3}^{\varepsilon}(t)\right|+\sqrt{\gamma}+\varepsilon^{2}\right) \\
& \leq \operatorname{const}\left(R, x_{0}, \xi_{0}, \delta\right)\left(\left|\eta_{1}^{\varepsilon}(t)\right|+\left|\tilde{\eta}_{2}^{\varepsilon}(t)\right|+\left|\eta_{3}^{\varepsilon}(t)\right|+\sqrt{\gamma}+\varepsilon^{2}\right) .
\end{aligned}
$$

It is readily verified that all the constants in the various estimates contained in the previous sections can be bounded from above by quantities which are independent upon $\varepsilon$. In turn, taking into account Lemma 3.5 and Proposition 3.7, there exists a positive constant $C$ such that

$\left|\eta_{1}^{\varepsilon}(t)\right|+\left|\tilde{\eta}_{2}^{\varepsilon}(t)\right|+\left|\eta_{3}^{\varepsilon}(t)\right| \leq C \varepsilon^{2}+C \int_{0}^{t}\left(\left|\eta_{1}^{\varepsilon}(\tau)\right|+\left|\tilde{\eta}_{2}^{\varepsilon}(\tau)\right|+\left|\eta_{3}^{\varepsilon}(\tau)\right|\right) d \tau, \quad$ for all $t \in\left[0, T^{\varepsilon, \gamma}\right)$.

Then, Gronwall lemma yields $\left|\eta_{1}^{\varepsilon}(t)\right|+\left|\tilde{\eta}_{2}^{\varepsilon}(t)\right|+\left|\eta_{3}^{\varepsilon}(t)\right| \leq C \varepsilon^{2}$ for all $t \in\left[0, T^{\varepsilon, \gamma}\right)$ and in turn also $\left|\eta_{1}^{\varepsilon}(t)\right|+\left|\eta_{2}^{\varepsilon}(t)\right|+\left|\eta_{3}^{\varepsilon}(t)\right| \leq C \varepsilon^{2}$ for all $t \in\left[0, T^{\varepsilon, \gamma}\right)$. Also from Lemma 3.4 , it holds $\varepsilon\left|w^{\varepsilon}(t)\right| \leq C \varepsilon^{2}$. In particular in (3.12) one can take $T^{\varepsilon, \gamma}=T$ for $\varepsilon$ small enough. Then, from Lemma 3.3 there exist functions $\theta^{\varepsilon}:\left[0, T^{\varepsilon, \gamma}\right) \rightarrow[0,2 \pi)$ such that

$$
\left\|u_{\varepsilon}(t, x)-e^{\frac{2}{\varepsilon}\left(\xi(t) \cdot x+\theta^{\varepsilon}(t)\right)} R\left(\frac{x-x^{\varepsilon}(t)}{\varepsilon}\right)\right\|_{H_{\varepsilon}^{1}}^{2} \leq C \varepsilon^{2}, \quad \text { for all } t \in[0, T]
$$

which together with

$$
\left\|R\left(\frac{x-x^{\varepsilon}(t)}{\varepsilon}\right)-R\left(\frac{x-x(t)}{\varepsilon}\right)\right\|_{H^{1}}^{2} \leq\left|w^{\varepsilon}\right|^{2}\|\nabla R\|_{H^{1}}^{2} \leq C \varepsilon^{2}
$$

concludes the proof of Theorem 1.1.

\section{Appendix A. Semi-singular potentials}

Let $\varepsilon, \delta \in(0,1], N \geq 1$ and $0<p<2 / N$. In this section, we shall consider the nonlinear Schrödinger equation for a family of smooth nearly singular external potentials $V_{\delta}: \mathbb{R}^{N} \rightarrow \mathbb{R}$,

$$
\imath \partial_{t} u+\frac{\varepsilon^{2}}{2} \Delta u-V_{\delta}(x) u+|u|^{2 p} u=0, \quad t \in \mathbb{R}, x \in \mathbb{R}^{N},
$$


where $\imath$ is the imaginary unit and $u: \mathbb{R} \times \mathbb{R}^{N} \rightarrow \mathbb{C}$ is a complex-valued function. we want to investigate the soliton dynamics behavior as $\varepsilon \rightarrow 0$ of the solutions to (A.1) which start from a rescaled bump-like initial data of the form

$$
u(x, 0)=R\left(\frac{x-x_{0}}{\varepsilon}\right) e^{\frac{2}{\varepsilon} \xi_{0} \cdot x}, \quad x_{0}, \xi_{0} \in \mathbb{R}^{N},
$$

Consider, for each $\delta \in(0,1]$, the Newtonian system

$$
\left\{\begin{array}{l}
\dot{x}=\xi \\
\dot{\xi}=-\nabla V_{\delta}(x), \\
x(0)=x_{0}, \quad \xi(0)=\xi_{0} .
\end{array}\right.
$$

Under suitable assumptions on the potential $V_{\delta}$, for each $\delta \in(0,1]$, system (A.3) admits a unique global solution $t \mapsto\left(x_{\delta}(t), \xi_{\delta}(t)\right)$ and its associated Hamiltonian energy $\mathscr{H}_{\delta}(t)=\frac{1}{2}\left|\xi_{\delta}(t)\right|^{2}+V_{\delta}\left(x_{\delta}(t)\right), t \geq 0$, remains constant through the motion. Let $\left(V_{\delta}\right)_{\delta \in(0,1]}$ be a family of functions $V_{\delta} \in C^{3}\left(\mathbb{R}^{N}, \mathbb{R}^{+}\right)$such that $\left\|D^{\alpha} V_{\delta}\right\|_{L^{\infty}}<\infty$ for every $0 \leq|\alpha| \leq 3$ and all $\delta \in(0,1]$. We define the function $\phi:(0,1] \rightarrow(0, \infty)$ by setting

$$
\phi(\delta):=\sum_{0 \leq|\alpha| \leq 3}\left\|D^{\alpha} V_{\delta}\right\|_{L^{\infty}}
$$

for all $\delta \in(0,1]$. We shall assume that there exists a set $\mathscr{V} \subset \mathbb{R}^{+} \times \mathbb{R}^{+}$such that $(0,0) \in \overline{\mathscr{V}}$ and

$$
\sup _{\substack{(\varepsilon, \delta) \in \mathscr{V} \\ \varepsilon, \delta \in(0,1]}} \varepsilon^{2} \phi(\delta)<+\infty, \quad \limsup _{\substack{(\varepsilon, \delta) \in \mathscr{Y} \\ \varepsilon \rightarrow 0^{+} \\ \delta \rightarrow 0^{+}}} \varepsilon^{2} \phi(\delta)=0 .
$$

Without loss of generality, we may assume that $\phi(\delta) \geq 1$, for all $\delta \in(0,1]$.

The main result of the Appendix, possibly useful for numerical purposes, is the following

Theorem A.1. Let $T>0$ and let $u^{\varepsilon, \delta}$ be the unique solution to problem (A.1)(A.2). Assume (A.4) and that for the initial position $x_{0} \in \mathbb{R}^{N}$ it holds

$$
\sup _{\delta \in(0,1]} V_{\delta}\left(x_{0}\right)<+\infty .
$$

Then there exist $C>0$, and $\varepsilon_{0}, \delta_{0}>0$ sufficiently small that

$$
u^{\varepsilon, \delta}(t, x)=R\left(\frac{\cdot-x_{\delta}(t)}{\varepsilon}\right) e^{\frac{2}{\varepsilon}\left(\xi_{\delta}(t) \cdot x+\vartheta^{\varepsilon, \delta}(t)\right)}+E^{\varepsilon, \delta}(x, t),\left\|E^{\varepsilon, \delta}(t, \cdot)\right\|_{H_{\varepsilon}^{1}} \leq C \varepsilon \phi^{2}(\delta),
$$

uniformly on $[0, T]$ for all $(\varepsilon, \delta) \in \mathscr{V}$ such that $0<\varepsilon \leq \varepsilon_{0}$ and $0<\delta \leq \delta_{0}$, being $x_{\delta}(t)$ the solution to system (A.3) and $\vartheta^{\varepsilon, \delta}$ a suitable shift term. In particular, provided that

$$
\begin{aligned}
& \limsup _{\substack{(\varepsilon, \delta) \in \mathscr{V} \\
\varepsilon \rightarrow 0^{+} \\
\delta \rightarrow 0^{+}}} \varepsilon \phi^{2}(\delta)=0, \\
& \quad
\end{aligned}
$$

a soliton dynamic behavior occurs.

The theorem will be proved using essentially the arguments developed in $[\mathbf{8}, \mathbf{1 4}]$ and explicitly highlighting the dependence of the conclusions from the parameter $\delta$ ruling the degree of singularity of the potential. 
A.1. Preparatory results. It is known that the solution $u^{\varepsilon, \delta}$ to (A.1)-(A.2) exists for all times $t$ with $u^{\varepsilon, \delta}(t) \in H^{2}\left(\mathbb{R}^{N}\right)$ and has conserved quantities, the mass

$$
\frac{1}{\varepsilon^{N}} \int_{\mathbb{R}^{N}}\left|u^{\varepsilon, \delta}(t, x)\right|^{2}=\|R\|_{L^{2}}^{2}:=m
$$

independently of $\varepsilon, \delta \in(0,1]$, and the energy

$$
\begin{aligned}
E_{\varepsilon, \delta}(t) & =\frac{1}{2 \varepsilon^{N-2}} \int_{\mathbb{R}^{N}}\left|\nabla u^{\varepsilon, \delta}(t)\right|^{2}+\frac{1}{\varepsilon^{N}} \int_{\mathbb{R}^{N}} V_{\delta}(x)\left|u^{\varepsilon, \delta}(t)\right|^{2} \\
& -\frac{1}{\varepsilon^{N}(p+1)} \int_{\mathbb{R}^{N}}\left|u^{\varepsilon, \delta}(t)\right|^{2 p+2}=E_{\varepsilon, \delta}(0) .
\end{aligned}
$$

In the spirit of $[\mathbf{1 4}$, Lemma 3.3] it holds

Lemma A.2. There exists a positive constant $C$ such that

$$
\left|\int_{\mathbb{R}^{N}} V_{\delta}\left(x_{0}+\varepsilon x\right) R^{2}(x)-m V_{\delta}\left(x_{0}\right)\right| \leq C \varepsilon^{2} \phi(\delta), \forall x_{0} \in \mathbb{R}^{N}, \forall \varepsilon \in(0,1], \forall \delta \in(0,1] .
$$

Lemma A.3. Let $u^{\varepsilon, \delta}$ be the unique solution to (A.1)-(A.2). Assume that for the initial position $x_{0} \in \mathbb{R}^{N}$

$$
\sup _{\delta \in(0,1]} V_{\delta}\left(x_{0}\right)<+\infty
$$

There exists a positive constant $C$ such that

$$
\sup _{t \geq 0}\left\|\nabla u^{\varepsilon, \delta}(t)\right\|_{L^{2}}^{2} \leq C \varepsilon^{N-2}+C \varepsilon^{N} \phi(\delta), \quad \forall \varepsilon \in(0,1], \forall \delta \in(0,1] .
$$

In particular, in light of (A.4), there holds

$$
\sup _{(\varepsilon, \delta) \in \mathscr{V}} \sup _{t \geq 0} \varepsilon^{2-N}\left\|\nabla u^{\varepsilon, \delta}(t)\right\|_{L^{2}}^{2}<+\infty .
$$

Proof. Taking into account that $V_{\delta}(x) \geq 0$ for all $x \in \mathbb{R}^{N}$ and $\delta>0$, by the conservation of energy and using Lemma A.2 and assumption (A.6), it follows that

$$
\begin{aligned}
& \frac{1}{2 \varepsilon^{N-2}} \int_{\mathbb{R}^{N}}\left|\nabla u^{\varepsilon, \delta}(t)\right|^{2}-\frac{1}{\varepsilon^{N}(p+1)} \int_{\mathbb{R}^{N}}\left|u^{\varepsilon, \delta}(t)\right|^{2 p+2} \leq E_{\varepsilon, \delta}(0) \\
& =\frac{1}{2} \int_{\mathbb{R}^{N}}|\nabla R|^{2}+\int_{\mathbb{R}^{N}} V_{\delta}\left(x_{0}+\varepsilon x\right) R^{2}(x)-\frac{1}{p+1} \int_{\mathbb{R}^{N}} R^{2 p+2}(x) \\
& \leq \frac{1}{2} \int_{\mathbb{R}^{N}}|\nabla R|^{2}+m V_{\delta}\left(x_{0}\right)+C \varepsilon^{2} \phi(\delta)-\frac{1}{p+1} \int_{\mathbb{R}^{N}} R^{2 p+2}(x) \leq C+C \varepsilon^{2} \phi(\delta),
\end{aligned}
$$

yielding in turn

$$
\left\|\nabla u^{\varepsilon, \delta}(t)\right\|_{L^{2}}^{2} \leq C \varepsilon^{N-2}+C \varepsilon^{N} \phi(\delta)+\frac{C}{\varepsilon^{2}}\left\|u^{\varepsilon, \delta}(t)\right\|_{2 p+2}^{2 p+2},
$$

for all $t \in[0, \infty)$ and for any $\varepsilon \in(0,1]$ and $\delta \in(0,1]$. Set $\theta=N p /(2 p+2)$. By the conservation of mass (A.5) it holds $\left\|u^{\varepsilon, \delta}(t)\right\|_{L^{2}}=C \varepsilon^{N / 2}$ for all $t \in[0, \infty)$ and for any $\varepsilon, \delta \in(0,1]$. Then, by virtue of the Gagliardo-Nirenberg inequality, it follows $\left\|u^{\varepsilon, \delta}(t)\right\|_{2 p+2} \leq C \varepsilon^{(1-\theta) N / 2}\left\|\nabla u^{\varepsilon, \delta}(t)\right\|_{L^{2}}^{\theta}$ for all $t \in[0, \infty)$ and any $\varepsilon, \delta \in(0,1]$. By the definition of $\theta$ and Young's inequality we reach

$$
\frac{C}{\varepsilon^{2}}\left\|u^{\varepsilon, \delta}(t)\right\|_{2 p+2}^{2 p+2} \leq C \varepsilon^{N-2}+\frac{1}{2}\left\|\nabla u^{\varepsilon, \delta}(t)\right\|_{L^{2}}^{2},
$$

for all $t \geq 0$, which immediately concludes the proof. 
The solution $u^{\varepsilon, \delta}$ enjoys the following energy splitting.

Lemma A.4. Let $u^{\varepsilon, \delta}$ be the unique solution to (A.1)-(A.2). There exists a positive constant $C$ such that

$$
E_{\varepsilon, \delta}\left(u^{\varepsilon, \delta}(t)\right)=\mathscr{E}(R)+m \mathscr{H}_{\delta}(t)+C \varepsilon^{2} \phi(\delta),
$$

for all $t \in[0, \infty)$ and for any $\varepsilon \in(0,1]$ and $\delta \in(0,1]$.

Proof. It is sufficient to observe that, by the conservation of energies $E_{\varepsilon, \delta}$ and $\mathscr{H}_{\delta}$ and taking into account Lemma A.2, we obtain

$E_{\varepsilon, \delta}\left(u^{\varepsilon, \delta}(t)\right)=\frac{1}{2} m\left|\xi_{0}\right|^{2}+m V_{\delta}\left(x_{0}\right)+\mathscr{E}(R)+C \varepsilon^{2} \phi(\delta)=m \mathscr{H}_{\delta}(t)+\mathscr{E}(R)+C \varepsilon^{2} \phi(\delta)$, for all $t \in[0, \infty)$ and for any $\varepsilon \in(0,1]$ and $\delta \in(0,1]$.

Following [14], let us now consider the auxiliary function

$$
\Psi_{\varepsilon, \delta}(t, x):=e^{-\frac{2}{\varepsilon}\left(\varepsilon x+x_{\delta}(t)\right) \cdot \xi_{\delta}(t)} u^{\varepsilon, \delta}\left(\varepsilon x+x_{\delta}(t)\right) .
$$

It is readily seen that $\left\|\Psi_{\varepsilon, \delta}(t)\right\|_{L^{2}}^{2}=\|R\|_{L^{2}}^{2}$ for every $t \geq 0$ and

$\mathscr{E}\left(\Psi_{\varepsilon, \delta}(t)\right)=E_{\varepsilon, \delta}\left(u^{\varepsilon, \delta}(t)\right)-\frac{1}{\varepsilon^{N}} \int_{\mathbb{R}^{N}} V_{\delta}(x)\left|u^{\varepsilon, \delta}\right|^{2}+\frac{1}{2} m\left|\xi_{\delta}(t)\right|^{2}-\xi_{\delta}(t) \cdot \int_{\mathbb{R}^{N}} p_{\varepsilon, \delta}(t, x)$, where $p_{\varepsilon, \delta}: \mathbb{R} \times \mathbb{R}^{N} \rightarrow \mathbb{R}^{N}$ is the momentum defined by

$$
p_{\varepsilon, \delta}(t, x):=\frac{1}{\varepsilon^{N-1}} \Im\left(\bar{u}^{\varepsilon, \delta}(t, x) \nabla u^{\varepsilon, \delta}(t, x)\right), \quad t \in \mathbb{R}, x \in \mathbb{R}^{N} .
$$

Lemma A.5. There exists a positive constant $C$ such that

$$
\left|\int_{\mathbb{R}^{N}} p_{\varepsilon, \delta}(t, x)\right| \leq C+C \varepsilon \sqrt{\phi(\delta)}, \quad \forall t \in[0, \infty), \forall \varepsilon \in(0,1], \forall \delta \in(0,1] .
$$

In particular, in light of (A.4), there holds

$$
\sup _{(\varepsilon, \delta) \in \mathscr{V}} \sup _{t \geq 0}\left|\int_{\mathbb{R}^{N}} p_{\varepsilon, \delta}(t, x)\right|<+\infty .
$$

Proof. Taking into account (A.5), by Hölder inequality we get

$$
\begin{aligned}
& \left|\int_{\mathbb{R}^{N}} p_{\varepsilon, \delta}(t, x)\right| \leq \int_{\mathbb{R}^{N}} \frac{\left|u^{\varepsilon, \delta}(t, x)\right|}{\varepsilon^{N / 2}} \frac{\left|\nabla u^{\varepsilon, \delta}(t, x)\right|}{\varepsilon^{N / 2-1}} \\
& \quad \leq C \varepsilon^{\frac{2-N}{2}}\left\|\nabla u^{\varepsilon, \delta}(t)\right\|_{L^{2}} \leq C+C \varepsilon \sqrt{\phi(\delta)} .
\end{aligned}
$$

for all $t \in[0, \infty)$ and for any $\varepsilon \in(0,1]$ and $\delta \in(0,1]$. Then, Lemma A.3 yields the assertion.

Lemma A.6. Assume that (A.6) holds. Then, there holds

$$
\sup _{\delta \in(0,1]} \sup _{t \geq 0}\left|\xi_{\delta}(t)\right|<+\infty .
$$

Proof. Since the energy functional $\mathscr{H}_{\delta}(t)=\frac{1}{2}\left|\xi_{\delta}(t)\right|^{2}+V_{\delta}\left(x_{\delta}(t)\right)$ associated with (A.3) remains constant, for any $t \geq 0$, taking into account that $V_{\delta} \geq 0$, there holds

$$
\left|\xi_{\delta}(t)\right|^{2}=2 \mathscr{H}_{\delta}(t)-2 V_{\delta}\left(x_{\delta}(t)\right) \leq 2 \mathscr{H}_{\delta}(t)=2 \mathscr{H}_{\delta}(0)=\left|\xi_{0}\right|^{2}+V_{\delta}\left(x_{0}\right) \leq C,
$$

where the last bound is due to (A.6). This proves the assertion. 
Lemma A.7. Assume that (A.6) holds. Then

$$
\sup _{\delta \in(0,1]} \sup _{t \in\left[0, \phi(\delta)^{-1}\right]}\left|x_{\delta}(t)\right|<+\infty .
$$

Proof. In light of Lemma A.6 and since $\phi(\delta) \geq 1$ there holds

$$
\left|x_{\delta}(t)\right| \leq\left|x_{0}\right|+\int_{0}^{t}\left|\xi_{\delta}(s)\right| d s \leq C+C t \leq C+\frac{C}{\phi(\delta)} \leq C
$$

for all $\delta \in(0,1]$ and $t \in\left[0, \phi(\delta)^{-1}\right]$, yielding the assertion.

We now recall $[\mathbf{1 4}$, Lemma 3.2] the following

LEMmA A.8. There exist $C_{0}>1$ and $K_{0}>0$ with $\left|\xi_{2}-\xi_{1}\right| \leq C_{0}\left\|\delta_{\xi_{2}}-\delta_{\xi_{1}}\right\|_{C^{2 *}}$ if $\left\|\delta_{\xi_{2}}-\delta_{\xi_{1}}\right\|_{C^{2 *}} \leq K_{0}$.

On account of (A.9) and Lemma A.4, for the family $\Psi_{\varepsilon, \delta}$ we have the following energy splitting

$$
\begin{gathered}
\mathcal{E}\left(\Psi^{\varepsilon, \delta}(t)\right)-\mathscr{E}(R)=\xi_{\delta}(t) \cdot\left(m \xi_{\delta}(t)-\int_{\mathbb{R}^{N}} p_{\varepsilon, \delta}(t, x)\right)+m V_{\delta}\left(x_{\delta}(t)\right) \\
-\frac{1}{\varepsilon^{N}} \int_{\mathbb{R}^{N}} V_{\delta}(x)\left|u^{\varepsilon, \delta}(t, x)\right|^{2}+C \varepsilon^{2} \phi(\delta),
\end{gathered}
$$

for all $t \in[0, \infty)$ and for any $\varepsilon \in(0,1]$ and $\delta \in(0,1]$. We shall now set

$$
\eta_{1}^{\varepsilon, \delta}(t):=m \xi_{\delta}(t)-\int_{\mathbb{R}^{N}} p_{\varepsilon, \delta}(t, x), \eta_{2}^{\varepsilon, \delta}(t):=m V_{\delta}\left(x_{\delta}(t)\right)-\frac{1}{\varepsilon^{N}} \int_{\mathbb{R}^{N}} V_{\delta}(x)\left|u^{\varepsilon, \delta}(t, x)\right|^{2},
$$

Furthermore, if $C_{0}, K_{0}$ are the constants in Lemma A.8, let us set

$$
M:=\sup _{\delta \in(0,1]} \sup _{t \in\left[0, \phi(\delta)^{-1}\right]} C_{0}\left|x_{\delta}(t)\right|+C_{0} K_{0} .
$$

In light of Lemma A.7, $M>0$ is finite. Of course $\left|x_{\delta}(t)\right| \leq M$, for every $\delta \in(0,1]$ and $t \in\left[0, \phi(\delta)^{-1}\right]$. We denote by $\chi$ a cut-off function such that $\chi=1$ on $|x| \leq M$ and $\chi=0$ on $|x| \geq 2 M$. Finally, also set

$$
\eta_{3}^{\varepsilon, \delta}(t):=m x_{\delta}(t)-\frac{1}{\varepsilon^{N}} \int_{\mathbb{R}^{N}} x \chi(x)\left|u^{\varepsilon, \delta}(t, x)\right|^{2},
$$

for every $t \geq 0$. Taking into account Lemma A.6, we finally achieve the following

LEMmA A.9. Let $u^{\varepsilon, \delta}$ be the unique solution to problem (A.1)-(A.2) and let $\Psi^{\varepsilon, \delta}$ the function defined in formula (A.8). Furthermore, let us set $\eta^{\varepsilon, \delta}(t)=\left|\eta_{1}^{\varepsilon, \delta}(t)\right|+$ $\left|\eta_{2}^{\varepsilon, \delta}(t)\right|+\left|\eta_{3}^{\varepsilon, \delta}(t)\right|$. Then there exists a positive constant $C$ such that $0 \leq \mathcal{E}\left(\Psi^{\varepsilon, \delta}(t)\right)-$ $\mathscr{E}(R) \leq C \eta^{\varepsilon, \delta}(t)+C \varepsilon^{2} \phi(\delta)$, for every $t \geq 0$.

LemmA A.10. The functions $\eta_{i}^{\varepsilon, \delta}:[0, \infty) \rightarrow \mathbb{R}, i=1,2,3$ are continuous and

$$
\eta_{1}^{\varepsilon, \delta}(0)=0, \quad\left|\eta_{2}^{\varepsilon, \delta}(0)\right| \leq C \varepsilon^{2} \phi(\delta), \quad\left|\eta_{3}^{\varepsilon, \delta}(0)\right| \leq C \varepsilon^{2},
$$

for some $C>0$ and for any $\varepsilon \in(0,1]$ and $\delta \in(0,1]$.

Proof. We easily get $\eta_{1}^{\varepsilon, \delta}(0)=m \xi_{0}-\int_{\mathbb{R}^{N}} p_{\varepsilon, \delta}(0, x)=m \xi_{0}-\xi_{0} \int_{\mathbb{R}^{N}} R^{2}(x)=0$. Moreover, we have

$$
\left|\eta_{2}^{\varepsilon, \delta}(0)\right|=\left|m V_{\delta}\left(x_{0}\right)-\int_{\mathbb{R}^{N}} V_{\delta}\left(x_{0}+\varepsilon x\right) R^{2}\right| \leq C \varepsilon^{2} \phi(\delta),\left|\eta_{3}^{\varepsilon, \delta}(0)\right|
$$




$$
=\left|m x_{0}-\int_{\mathbb{R}^{N}}\left(x_{0}+\varepsilon x\right) \chi\left(x_{0}+\varepsilon x\right) R^{2}\right| \leq C \varepsilon^{2},
$$

in light of Lemma A.2.

Let us introduce the time

$$
T^{\varepsilon, \delta}:=\sup \left\{t \in\left[0, \phi(\delta)^{-1}\right]: \eta^{\varepsilon, \delta}(s) \leq \mu, \text { for all } s \in(0, t)\right\},
$$

where, recalling (A.4), $\mu>0$ is a sufficiently small positive constant such that

$$
\left\{\begin{array}{l}
C \eta^{\varepsilon, \delta}(t)+C \varepsilon^{2} \phi(\delta) \leq \mathcal{A}, \\
\text { for every } t \in\left[0, T^{\varepsilon, \delta}\right) \text { and all }(\varepsilon, \delta) \in \mathscr{V} \text { such that } \\
0<\varepsilon \leq \varepsilon_{0} \text { and } 0<\delta \leq \delta_{0}, \text { where } \varepsilon_{0}, \delta_{0} \text { are small enough. }
\end{array}\right.
$$

being $\mathcal{A}>0$ the constant which appears in the statement of Proposition 3.2 and $C>0$ is the constant which appears in the statement of Lemma A.9.

In this framework, by virtue of Proposition 3.2, we find families of functions $\theta^{\varepsilon, \delta}$ : $\left[0, T^{\varepsilon, \delta}\right) \rightarrow[0,2 \pi)$ and $\xi^{\varepsilon, \delta}:\left[0, T^{\varepsilon, \delta}\right) \rightarrow \mathbb{R}^{N}$ such that

$$
\left\|\Psi^{\varepsilon, \delta}(t)-e^{\imath \theta^{\varepsilon, \delta}} R\left(\cdot-\xi^{\varepsilon, \delta}\right)\right\|_{H^{1}}^{2} \leq C \eta^{\varepsilon, \delta}(t)+C \varepsilon^{2} \phi(\delta)
$$

for all $t \in\left[0, T^{\varepsilon, \delta}\right)$. Then, we get the following

Lemma A.11. There exist families of functions $\theta^{\varepsilon, \delta}:\left[0, T^{\varepsilon, \delta}\right) \rightarrow[0,2 \pi)$ and $\xi^{\varepsilon, \delta}:\left[0, T^{\varepsilon, \delta}\right) \rightarrow \mathbb{R}^{N}$ with

$$
\left\|u^{\varepsilon, \delta}(t)-e^{\frac{2}{\varepsilon}\left(\xi_{\delta}(t) \cdot x+\vartheta^{\varepsilon, \delta}(t)\right)} R\left(\frac{\cdot-x_{\delta}(t)}{\varepsilon}+\xi^{\varepsilon, \delta}\right)\right\|_{H_{\varepsilon}^{1}}^{2} \leq C \eta^{\varepsilon, \delta}(t)+C \varepsilon^{2} \phi(\delta),
$$

for all $t \in\left[0, T^{\varepsilon, \delta}\right)$, where $w^{\varepsilon, \delta}:=x_{\delta}(t)-\varepsilon \xi^{\varepsilon, \delta}$ and $\vartheta^{\varepsilon, \delta}(t):=\varepsilon \theta^{\varepsilon, \delta}(t)$.

We now aim to prove the following

Lemma A.12. Let $u^{\varepsilon, \delta}$ be the unique solution to (A.1)-(A.2). Then there exists a positive constant $C$ with

$\left\|\varepsilon^{-N} u^{\varepsilon, \delta}(\cdot, t)-m \delta_{x_{\delta}(t)}\right\|_{C^{2 *}}+\left\|p^{\varepsilon, \delta}(\cdot, t) d x-m \xi_{\delta}(t) \delta_{x_{\delta}(t)}\right\|_{C^{2 *}} \leq C \eta^{\varepsilon, \delta}(t)+C \varepsilon^{2} \phi(\delta)$, for every $t \in\left[0, T^{\varepsilon, \delta}\right)$ and all $(\varepsilon, \delta) \in \mathscr{V}$ such that $0<\varepsilon \leq \varepsilon_{0}$ and $0<\delta \leq \delta_{0}$.

Proof. Let $u^{\varepsilon, \delta}$ be the unique solution to problem (A.1)-(A.2). Then, in the spirit of [14, Lemma 3.4], it is possible to prove that there exists a positive constant $C$, independent of $\varepsilon$ and $\delta$, such that

(A.13)

$\left\|\varepsilon^{-N} u^{\varepsilon, \delta}(\cdot, t) d x-m \delta_{w^{\varepsilon, \delta}(t)}\right\|_{C^{2 *}}+\left\|p^{\varepsilon, \delta}(\cdot, t) d x-m \xi_{\delta}(t) \delta_{w^{\varepsilon, \delta}(t)}\right\|_{C^{2 *}} \leq C \eta^{\varepsilon, \delta}(t)+C \varepsilon^{2} \phi(\delta)$,

for every $t \in\left[0, T^{\varepsilon, \delta}\right)$ and all $(\varepsilon, \delta) \in \mathscr{V}$ such that $0<\varepsilon \leq \varepsilon_{0}$ and $0<\delta \leq \delta_{0}$. Let us now prove that there exists $\mu>0$ and a positive constant $C$, independent of $\varepsilon$ and $\delta$, such that

$$
\left|x_{\delta}(t)-w^{\varepsilon, \delta}(t)\right| \leq C \eta^{\varepsilon, \delta}(t)+C \varepsilon^{2} \phi(\delta),
$$

for every $t \in\left[0, T^{\varepsilon, \delta}\right)$ and all $(\varepsilon, \delta) \in \mathscr{V}$ such that $0<\varepsilon \leq \varepsilon_{0}$ and $0<\delta \leq \delta_{0}$. We follow the proof of [14, Lemma 3.5]. Assuming that $\left|w^{\varepsilon, \delta}(t)\right| \leq M$ for every $t \in\left[0, T^{\varepsilon, \delta}\right)$ and all $(\varepsilon, \delta) \in \mathscr{V}$ such that $0<\varepsilon \leq \varepsilon_{0}$ and $0<\bar{\delta} \leq \delta_{0}$ (up to 
further reducing the size of $\delta_{0}$ ), the assertion follows, since by the definition of $\chi$ and (A.13),

$$
\begin{aligned}
\left|x_{\delta}(t)-w^{\varepsilon, \delta}(t)\right| & \leq \frac{1}{m}\left|\int_{\mathbb{R}^{N}} x \chi(x) \frac{\left|u^{\varepsilon, \delta}(t, x)\right|^{2}}{\varepsilon^{N}}-m w^{\varepsilon, \delta}(t)\right|+\frac{1}{m} \eta^{\varepsilon, \delta}(t) \\
& \leq C\|x \chi\|_{C^{2}}\left\|\varepsilon^{-N} u^{\varepsilon, \delta}(\cdot, t) d x-m \delta_{w^{\varepsilon, \delta}(t)}\right\|_{C^{2 *}}+C \eta^{\varepsilon, \delta}(t) \\
& \leq C \eta^{\varepsilon, \delta}(t)+C \varepsilon^{2} \phi(\delta),
\end{aligned}
$$

for every $t \in\left[0, T^{\varepsilon, \delta}\right)$ and all $(\varepsilon, \delta) \in \mathscr{V}$ such that $0<\varepsilon \leq \varepsilon_{0}$ and $0<\delta \leq \delta_{0}$. Thus, it is left to show that $\left|w^{\varepsilon, \delta}(t)\right| \leq M$ for all $t \in\left[0, T^{\varepsilon, \delta}\right)$ and $(\varepsilon, \delta) \in \mathscr{V}$ such that $0<\varepsilon \leq \varepsilon_{0}$ and $0<\delta \leq \delta_{0}$, up to further reducing the size of $\delta_{0}$. On account of Lemma A.5, and arguing as in $[\mathbf{1 4}$, p.183] there exists a constant $C$, independent of $\varepsilon$ and $\delta$, such that for all $t_{1}, t_{2} \in\left[0, T^{\varepsilon, \delta}\right)$ with $t_{1}<t_{2}$

$$
\left\|\varepsilon^{-N} u^{\varepsilon, \delta}\left(\cdot, t_{2}\right) d x-\varepsilon^{-N} u^{\varepsilon, \delta}\left(\cdot, t_{1}\right) d x\right\|_{C^{2 *}} \leq C\left|t_{2}-t_{1}\right| \leq \frac{2 C}{\phi(\delta)},
$$

yielding in turn by (A.13) and the definition (A.11)-(A.12) of $T^{\varepsilon, \delta}$

$$
\begin{gathered}
\left\|m \delta_{w^{\varepsilon, \delta}\left(t_{2}\right)}-m \delta_{w^{\varepsilon, \delta}\left(t_{1}\right)}\right\|_{C^{2 *}} \leq C\left[\eta^{\varepsilon, \delta}\left(t_{2}\right)+\eta^{\varepsilon, \delta}\left(t_{1}\right)+\varepsilon^{2} \phi(\delta)+\frac{1}{\phi(\delta)}\right] \\
\leq C \mu+C \varepsilon^{2} \phi(\delta)+\frac{C}{\phi(\delta)} .
\end{gathered}
$$

Therefore, up to reducing the value of $\delta_{0}$, choosing $\mu>0$ sufficiently small in the definition of $T^{\varepsilon, \delta}$, we have $\left\|\delta_{w^{\varepsilon, \delta}\left(t_{2}\right)}-\delta_{w^{\varepsilon, \delta}\left(t_{1}\right)}\right\|_{C^{2 *}} \leq K_{0}$ for every $t \in\left[0, T^{\varepsilon, \delta}\right)$ and all $(\varepsilon, \delta) \in \mathscr{V}$ such that $0<\varepsilon \leq \varepsilon_{0}$ and $0<\delta \leq \delta_{0}$, where $K_{0}$ is the constant appearing in the statement of Lemma A.8. By virtue of Lemma A.8, it holds $\left|w^{\varepsilon, \delta}\left(t_{2}\right)-w^{\varepsilon, \delta}\left(t_{1}\right)\right| \leq C_{0}\left\|\delta_{w^{\varepsilon, \delta}\left(t_{2}\right)}-\delta_{w^{\varepsilon, \delta}\left(t_{1}\right)}\right\|_{C^{2 *}} \leq C_{0} K_{0}$. Since $w^{\varepsilon, \delta}(0)=x_{0}$, it follows $\left|w^{\varepsilon, \delta}(t)\right| \leq C_{0} K_{0}+\left|x_{0}\right| \leq M$, yielding the desired conclusion. As a consequence of (A.14), there holds $\left\|\delta_{x_{\delta}(t)}-\delta_{w^{\varepsilon, \delta}(t)}\right\|_{C^{2 *}} \leq\left|x_{\delta}(t)-w^{\varepsilon, \delta}(t)\right| \leq C \eta^{\varepsilon, \delta}(t)+C \varepsilon^{2} \phi(\delta)$, for all $t \in\left[0, T^{\varepsilon, \delta}\right)$ and all $(\varepsilon, \delta) \in \mathscr{V}$ such that $0<\varepsilon \leq \varepsilon_{0}$ and $0<\delta \leq \delta_{0}$. This yields the assertion by (A.13).

Lemma A.13. $\eta^{\varepsilon, \delta}(t) \leq C \varepsilon^{2} \phi(\delta)$ for all $t \in\left[0, T^{\varepsilon, \delta}\right)$ and all $(\varepsilon, \delta) \in \mathscr{V}$ with $0<\varepsilon \leq \varepsilon_{0}$ and $0<\delta \leq \delta_{0}$.

Proof. We have

$$
\eta^{\varepsilon, \delta}(t) \leq C \varepsilon^{2} \phi(\delta)+\int_{0}^{t} \sum_{j=1}^{3}\left|\frac{d}{d t} \eta_{j}^{\varepsilon, \delta}(\sigma)\right| d \sigma
$$

We recall that, as known, the following identities holds

$$
\int_{\mathbb{R}^{N}} \frac{\partial}{\partial t} p^{\varepsilon, \delta}(t, x)=\frac{1}{\varepsilon^{N}} \int_{\mathbb{R}^{N}} \nabla V_{\delta}(x)\left|u^{\varepsilon, \delta}(t, x)\right|^{2}, \frac{\partial}{\partial t} \frac{\left|u^{\varepsilon, \delta}(t, x)\right|^{2}}{\varepsilon^{N}}=-\operatorname{div}_{x} p^{\varepsilon, \delta}(t, x) .
$$

In turn, by Lemma A.12, we have

$$
\begin{aligned}
\left|\frac{d}{d t} \eta_{1}^{\varepsilon, \delta}(t)\right| & =\left.\left|m \dot{\xi}_{\delta}(t)+\frac{1}{\varepsilon^{N}} \int_{\mathbb{R}^{N}} \nabla V_{\delta}(x)\right| u^{\varepsilon, \delta}(t, x)\right|^{2} \mid \\
& \leq\left\|\nabla V_{\delta}\right\|_{C^{2}}\|\| \varepsilon^{-N} u^{\varepsilon, \delta}(\cdot, t) d x-m \delta_{x_{\delta}(t)} \|_{C^{2 *}} \leq C \phi(\delta) \eta^{\varepsilon, \delta}(t)+C \varepsilon^{2} \phi^{2}(\delta),
\end{aligned}
$$


for every $t \in\left[0, T^{\varepsilon, \delta}\right)$ and all $(\varepsilon, \delta) \in \mathscr{V}$ such that $0<\varepsilon \leq \varepsilon_{0}$ and $0<\delta \leq \delta_{0}$. Then,

$$
\begin{gathered}
\int_{0}^{t}\left|\frac{d}{d t} \eta_{1}^{\varepsilon, \delta}(\sigma)\right| d \sigma \leq C \phi(\delta) \int_{0}^{t} \eta^{\varepsilon, \delta}(\sigma) d \sigma \\
+\int_{0}^{t} C \varepsilon^{2} \phi^{2}(\delta) \leq C \phi(\delta) \int_{0}^{t} \eta^{\varepsilon, \delta}(\sigma) d \sigma+C \varepsilon^{2} \phi(\delta),
\end{gathered}
$$

since $t \leq T^{\varepsilon, \delta} \leq \phi(\delta)^{-1}$. Analogously, again by Lemma A.12, we have

$$
\begin{aligned}
\left|\frac{d}{d t} \eta_{2}^{\varepsilon, \delta}(t)\right| & =\left|\nabla V_{\delta}\left(x_{\delta}(t)\right) \cdot m \xi_{\delta}(t)-\int_{\mathbb{R}^{N}} \nabla V_{\delta}(x) \cdot p^{\varepsilon, \delta}(t, x)\right| \\
& \leq\left\|\nabla V_{\delta}\right\|_{C^{2}}\|\| p^{\varepsilon, \delta}(\cdot, t) d x-m \xi_{\delta}(t) \delta_{x_{\delta}(t)} \|_{C^{2 *}} \leq C \phi(\delta) \eta^{\varepsilon, \delta}(t)+C \varepsilon^{2} \phi^{2}(\delta) .
\end{aligned}
$$

Then, as $t \leq T^{\varepsilon, \delta} \leq \phi(\delta)^{-1}$, we achieve

$$
\begin{gathered}
\int_{0}^{t}\left|\frac{d}{d t} \eta_{2}^{\varepsilon, \delta}(\sigma)\right| d \sigma \leq C \phi(\delta) \int_{0}^{t} \eta^{\varepsilon, \delta}(\sigma) d \sigma+\int_{0}^{t} C \varepsilon^{2} \phi^{2}(\delta) \\
\leq C \phi(\delta) \int_{0}^{t} \eta^{\varepsilon, \delta}(\sigma) d \sigma+C \varepsilon^{2} \phi(\delta) .
\end{gathered}
$$

The treatment of the term $\eta_{3}^{\varepsilon, \delta}$ follows as in the proof of [14, Lemma 3.6] yielding, as $t \leq T^{\varepsilon, \delta} \leq \phi(\delta)^{-1}$,

$$
\int_{0}^{t}\left|\frac{d}{d t} \eta_{3}^{\varepsilon, \delta}(\sigma)\right| d \sigma \leq C \int_{0}^{t} \eta^{\varepsilon, \delta}(\sigma) d \sigma+C \varepsilon^{2} \leq C \phi(\delta) \int_{0}^{t} \eta^{\varepsilon, \delta}(\sigma) d \sigma+C \varepsilon^{2} \phi(\delta) .
$$

Hence, by recollecting the previous inequalities, by virtue of Gronwall lemma and $t \leq T^{\varepsilon, \delta} \leq \phi(\delta)^{-1}$, it follows $\eta^{\varepsilon, \delta}(t) \leq C \varepsilon^{2} \phi(\delta) e^{\phi(\delta) t} \leq C \varepsilon^{2} \phi(\delta)$, concluding the proof.

A.2. Proof of Theorem A.1. By Lemma A.13 and the continuity of $\eta^{\varepsilon, \delta}$, it follows $T^{\varepsilon, \delta}=\phi(\delta)^{-1}$, yielding $\eta^{\varepsilon, \delta}(t) \leq C \varepsilon^{2} \phi(\delta)$, for every $t \in\left[0, \phi(\delta)^{-1}\right)$ and all $(\varepsilon, \delta) \in \mathscr{V}$ such that $0<\varepsilon \leq \varepsilon_{0}$ and $0<\delta \leq \delta_{0}$, up to reducing the value of $\varepsilon_{0}$ and $\delta_{0}$. Hence,

$$
\left\|u^{\varepsilon, \delta}(t)-e^{\frac{\imath}{\varepsilon}\left(\xi_{\delta}(t) \cdot x+\vartheta^{\varepsilon, \delta}(t)\right)} R\left(\frac{\cdot-x_{\delta}(t)}{\varepsilon}+\xi^{\varepsilon, \delta}\right)\right\|_{H_{\varepsilon}^{1}}^{2} \leq C \varepsilon^{2} \phi(\delta),
$$

for all $t \in\left[0, \phi(\delta)^{-1}\right)$ and all $(\varepsilon, \delta) \in \mathscr{V}$ with $0<\varepsilon \leq \varepsilon_{0}$ and $0<\delta \leq \delta_{0}$. Recall now that, since $w^{\varepsilon, \delta}=x_{\delta}(t)-\varepsilon \xi^{\varepsilon, \delta}$, in light of (A.14), we obtain $\left|\xi^{\varepsilon, \delta}\right|^{2} \leq C \varepsilon^{2} \phi(\delta)^{2}$. Then, we can conclude that $\left\|R(\cdot)-R\left(\cdot-\xi^{\varepsilon, \delta}\right)\right\|_{H^{1}}^{2} \leq C\left|\xi^{\varepsilon, \delta}\right|^{2} \leq C \varepsilon^{2} \phi(\delta)^{2}$. This combined with (A.17) yields

$$
\left\|u^{\varepsilon, \delta}(t)-e^{\frac{\imath}{\varepsilon}\left(\xi_{\delta}(t) \cdot x+\vartheta^{\varepsilon, \delta}(t)\right)} R\left(\frac{\cdot-x_{\delta}(t)}{\varepsilon}\right)\right\|_{H_{\varepsilon}^{1}} \leq C \varepsilon \phi(\delta),
$$

for all $t \in\left[0, \phi(\delta)^{-1}\right)$ and all $(\varepsilon, \delta) \in \mathscr{V}$ with $0<\varepsilon \leq \varepsilon_{0}$ and $0<\delta \leq \delta_{0}$. Fixed $T>0$ and arguing as in [14], up to an error of size $\varepsilon \phi(\delta)$ in $H_{\varepsilon}^{1}$ one can repeat the argument on the time interval $\left[\phi(\delta)^{-1}, 2 \phi(\delta)^{-1}\right]$ and so on. To cover the entire interval $[0, T]$ one therefore needs to add $\phi(\delta)$-times an error of size $\varepsilon \phi(\delta)$ in $H_{\varepsilon}^{1}$, yielding an overall error $\varepsilon \phi^{2}(\delta)$ in $H_{\varepsilon}^{1}$, reaching the control

$$
\left\|u^{\varepsilon, \delta}(t)-e^{\frac{\imath}{\varepsilon}\left(\xi_{\delta}(t) \cdot x+\vartheta^{\varepsilon, \delta}(t)\right)} R\left(\frac{\cdot-x_{\delta}(t)}{\varepsilon}\right)\right\|_{H_{\varepsilon}^{1}} \leq C \varepsilon \phi^{2}(\delta),
$$


for all $t \in[0, T]$ and all $(\varepsilon, \delta) \in \mathscr{V}$ with $0<\varepsilon \leq \varepsilon_{0}$ and $0<\delta \leq \delta_{0}$. This concludes the proof.

\section{References}

[1] W.K. Abou Salem, Solitary wave dynamics in time-dependent potentials, J. Math. Phys. 49 (2008), 032101.

[2] W.K. Abou Salem, Effective dynamics of solitons in the presence of rough nonlinear perturbations, Nonlinearity 22 (2009), 747-763.

[3] W.K. Abou Salem, J. Fröhlich, I.M. Sigal, Colliding solitons for the nonlinear Schrödinger equation, Comm. Math. Phys. 291 (2009), 151-176.

[4] R. Adami, D. Noja, Stability and symmetry breaking bifurcation for the ground states of a NLS equation with a $\delta^{\prime}$ interaction, Comm. Math. Phys., in press

[5] A. Ambrosetti, M. Badiale, S. Cingolani, Semiclassical states on nonlinear Schrödinger equations, Arch. Rational Mech. Anal. 140 (1997), 285-300.

[6] V. Benci, M. Ghimenti, A.M. Micheletti, The nonlinear Schrödinger equation: solitons dynamics, J. Differential Equations 249 (2010), 3312-3341.

[7] V. Benci, M. Ghimenti, A.M. Micheletti, On the dynamics of solitons in the nonlinear Schrödinger equation, Arch. Ration. Mech. Anal. in press

[8] J. Bronski, R. Jerrard, Soliton dynamics in a potential, Math. Res. Letters 7 (2000), 329-342.

[9] T. Cazenave, "Semilinear Schrödinger Equations", Courant Lect. Notes Math., vol. 10, New York University Courant Institute of Mathematical Sciences, New York, 2003.

[10] T. Cazenave, P.L. Lions, Orbital stability of standing waves for some nonlinear Schrödinger equations, Comm. Math. Phys. 85 (1982), 549-561.

[11] J. Fröhlich, S. Gustafson S., B.L.G. Jonsson, I.M. Sigal, Solitary wave dynamics in an external potential, Comm. Math. Phys. 250 (2004), 613-642.

[12] J. Fröhlich, S. Gustafson, B.L.G. Jonsson, I.M. Sigal, Long time motion of NLS solitary waves in a confining potential, Annals Henri Poincare 7 (2006), 621-660.

[13] J. Fröhlich, T.-P. Tsai, H.-T. Yau, On the point-particle (Newtonian) limit of the nonlinear Hartree equation, Comm. Math. Phys. 225 (2002), 223-274.

[14] S. Keranan, Semiclassical limit for nonlinear Schrödinger equation with potential. II. Asymptot. Anal. 47 (2006), 171-186.

[15] M.K. Kwong, Uniqueness of positive solutions of $\Delta u-u+u^{p}=0$ in $\mathbb{R}^{n}$, Arch. Rational Mech. Anal. 105 (1989), 243-266.

[16] E. Montefusco, B. Pellacci, M. Squassina, Energy convexity estimates for non degenerate ground states of nonlinear 1D Schrodinger systems Commun. Pure Appl. Anal. 9 (2010), 867884.

[17] E. Montefusco, B. Pellacci, M. Squassina, Soliton dynamics for CNLS systems with potentials Asymptotic Anal. 66 (2010), 61-86.

[18] A. Selvitella, Asymptotic evolution for the semiclassical nonlinear Schrödinger equation in presence of electric and magnetic fields, J. Differential Equations 245 (2008), 2566-2584.

[19] M. SquAssina, Soliton dynamics for the nonlinear Schrodinger equation with magnetic field, Manuscripta Math. 130 (2009), 461-494.

[20] C. Sulem, P.L. Sulem, "The nonlinear Schrödinger equation. Self-focusing and wave collapse", Appl. Math. Sci, 139, Springer, New York, 1999.

[21] M.I. Weinstein, Modulational stability of ground state of nonlinear Schrödinger equations, SIAM J. Math. Anal. 16 (1985), 472-491.

[22] M.I. Weinstein, Lyapunov stability of ground states of nonlinear dispersive evolution equations, Comm. Pure Appl. Math. 39 (1986), 51-67. 
Dipartimento di Matematica Applicata, Università degli Studi di Pisa, Via F. BuonarROTI, 1/C, I-56127 PISA, ITALY

E-mail address: bonanno@mail.dm.unipi.it

Dipartimento di Matematica Applicata, Università degli Studi di Pisa, Via F. BuonarRoti, 1/C, I-56127 PisA, ItALY

E-mail address: ghimenti@mail.dm.unipi.it

Dipartimento di Informatica, Università degli Studi di Verona, Cá Vignal 2, Strada Le Grazie 15, I-37134 Verona, Italy

E-mail address: marco.squassina@univr.it 